This item was submitted to Loughborough's Research Repository by the author.

Items in Figshare are protected by copyright, with all rights reserved, unless otherwise indicated.

\title{
The interaction between environmental temperature and hypoxia on central and peripheral fatigue during high-intensity dynamic knee extension
}

\section{PLEASE CITE THE PUBLISHED VERSION}

http://dx.doi.org/10.1152/japplphysiol.00876.2015

\section{PUBLISHER}

(C) The American Physiological Society

\section{VERSION}

AM (Accepted Manuscript)

\section{PUBLISHER STATEMENT}

This work is made available according to the conditions of the Creative Commons Attribution-NonCommercialNoDerivatives 4.0 International (CC BY-NC-ND 4.0) licence. Full details of this licence are available at: https://creativecommons.org/licenses/by-nc-nd/4.0/

\section{LICENCE}

CC BY-NC-ND 4.0

\section{REPOSITORY RECORD}

Lloyd, Alex, Margherita Raccuglia, S.G. Hodder, and George Havenith. 2019. "The Interaction Between Environmental Temperature and Hypoxia on Central and Peripheral Fatigue During High-intensity Dynamic Knee Extension". figshare. https://hdl.handle.net/2134/20166. 
1 Title:

2 The interaction between environmental temperature and hypoxia on central and peripheral

3 fatigue during high-intensity dynamic knee extension

4

5 Authors:

$6 \quad$ Alex Lloyd

7 Margherita Raccuglia

8 Simon Hodder

9 George Havenith

Affiliation:

Environmental Ergonomics Research Centre, Loughborough University, Loughborough, UK

14 Running Head:

15 Fatigue in combined hypoxic and thermal stress

17 Corresponding Author:

18 George Havenith

19 Environmental Ergonomics Research Centre,

20 James France Bldg.,

21 Loughborough,

22 Leicestershire

$23 \quad$ UK

24 LE11 3TU

25 Email: G.Havenith@lboro.ac.uk

26

27

28 
30 This study investigated causative factors behind the expression of different interaction types during

31

32 exposure to multi-stressor environments. Neuromuscular fatigue rates and time to exhaustion (TTE) were investigated in active males $(\mathrm{n}=9)$ exposed to three climates $\left(5^{\circ} \mathrm{C}, 50 \%\right.$-rh $/ 23^{\circ} \mathrm{C}, 50 \%$ $\mathrm{rh} / 42^{\circ} \mathrm{C}, 70 \%$-rh $)$ at two inspired oxygen fractions $\left(0.209 / 0.125 \mathrm{~F}_{\mathrm{I}} \mathrm{O}_{2}\right.$; equivalent attitude $\left.=4100 \mathrm{~m}\right)$. After 40-mins rest in the environmental conditions, participants performed constant workload (highintensity) knee extension exercise until exhaustion, with brief assessments of neuromuscular function every 110-s. Independent exposure to cold, heat and hypoxia significantly $(\mathrm{p}<0.01)$ reduced TTE from thermoneutral-normoxia (reduction of 190, 405, 505-s from 915-s respectively). The TTE decrease was consistent with a faster rate of peripheral fatigue development $(\mathrm{p}<0.01)$ compared with thermoneutralnormoxia (increase of $1.6,3.1$ and $4.9 \% \cdot \mathrm{min}^{-1}$ from $4.1 \% \cdot \mathrm{min}^{-1}$ respectively). Combined exposure to hypoxic-cold resulted in an even greater TTE reduction (-589-seconds), likely due to an increase in the rate of peripheral fatigue development (increased by $7.6 \% \cdot \mathrm{min}^{-1}$ ), but this without significant interaction between stressors $(\mathrm{p}>0.198)$. In contrast, combined exposure to hypoxic-heat reduced TTE by $609-\mathrm{s}$, showing a significant antagonistic interaction $(\mathrm{p}=0.003)$ similarly supported by an increased rate of peripheral fatigue development (increased by $\left.8.3 \% \cdot \mathrm{min}^{-1}\right)$. A small decline $\left(<0.4 \% \cdot \mathrm{min}^{-1}\right.$ ) in voluntary muscle activation was only observed in thermoneutral-normoxia. In conclusion, interaction type is influenced by the impact magnitude of the individual stressors' effect on exercise capacity, whereby the greater the stressors impact, the greater the probability that one stressor will be abolished by the other. This indicates humans respond to severe and simultaneous physiological strains based on a 'worst strain takes precedence' principle.

1 New and Noteworthy: A novel principle of multifactorial integration is proposed; that the type of interaction between physiological stressors is influenced by the impact magnitude of individual stressors' effect on exercise capacity. Mild stressors add up, however the greater the stressors' impact, the greater the trend for one stressor to cancel out the other. This ultimately infers a maximum threshold for performance deterioration, whereby humans respond to severe and simultaneous strains based on a 'worst-strain takes precedence' principle. 
58 Keywords: combined stressors, central motor drive, high altitude, neuromuscular fatigue, thermal 59 stress.

\section{ABBREVIATIONS}

61 ANOVA: Analysis of variance

62 COLD: Cold-normoxic condition (used in figures and tables only)

$63 \mathrm{CV}$ : Coefficient of variation

$64 \quad \mathrm{~F}_{\mathrm{I}} \mathrm{O}_{2}$ : Fraction of inspired oxygen

65 FNS: Femoral nerve stimulation (twitch interpolation)

66 HEAT: Heated-normoxic condition (used in figures and tables only)

67 HR: Heart rate

68 HYP-COLD: Hypoxic- cold condition (used in figures and tables only)

69 HYP-NEU: Hypoxic- thermoneutral condition (used in figures and tables only)

70 HYP-HEAT: Hypoxic- heat condition (used in figures and tables only)

71 MVC: Maximal voluntary contraction

72 RFD: Resting twitch rate of force development

73 RFR: Resting twitch rate of relaxation

74 NEU: Thermoneutral-normoxic condition (used in figures and tables only)

$75 \mathrm{O}_{2}$ : Oxygen

76 PRE-REST: Pre-rest (used in figures and tables only)

77 POST-REST: Post-rest (used in figures and tables only)

$78 \mathrm{Q}_{\mathrm{tw}, \mathrm{pot}}$ : Resting potentiated twitch force (peripheral fatigue)

$79 \mathrm{Q}_{\mathrm{tw}, \text { sup }}$ : Superimposed twitch force

$80 \quad \mathrm{SpO}_{2}$ : Oxygen saturation of peripheral blood

$81 \mathrm{~T}_{\text {core }}$ : Core temperature

$82 \mathrm{~T}_{\text {env }}$ : Environmental temperature

$83 \mathrm{~T}_{\mathrm{m}}$ : Muscle temperature

$84 \mathrm{~T}_{\mathrm{sk}}$ : Skin temperature

85 EXH: Exhaustion through task failure or exercise intolerance (used in figures and tables only)

86 TTE: Time to exhaustion

87 VA: Voluntary activation

$88 \% \mathrm{VO}_{2 \max }$ : Relative percentage of maximal aerobic capacity 
92 A human's ability to sustain mechanical function - muscular force and power - over time is modulated 93 by numerous environmental factors, including both the oxygen $\left(\mathrm{O}_{2}\right)$ availability and the climate $(3,42$, 94 69). While these are widely studied as independent stressors, many real life applications generate hypoxic and thermal stressors in combination e.g. endurance exercise in cold mountainous areas, operating/ piloting unpressurised aircraft or in the use of hypoxic-heat as a training stimulus $(19,31)$. Research to date suggests many of the key physiological strains associated with thermal (cold and heat) and hypoxic stress are precursors of a given mechanical work being performed at a higher absolute and/or relative aerobic strain i.e. increases in $\mathrm{ml} \mathrm{O}_{2} \cdot \mathrm{min}^{-1} \cdot \mathrm{W}^{-1}$ or $\% \mathrm{VO}_{2 \max } \cdot \mathrm{W}^{-1}$ respectively $(4,35,42)$. For example, previous studies have reported that during exhaustive or high intensity exercise in the heat $(20,40,50)$, the thermoregulatory requirements for skin blood flow, together with progressive dehydration and higher muscle sympathetic nerve activity, may compromise perfusion of (i.e. oxygen transport to) the active muscle $(21,55,57,60)$. This is similar to hypoxia, where a systemic reduction in arterial oxygen content strains the cardiovascular system's ability to meet the required oxygen delivery to active musculature $(4,15)$. As such, both heat and hypoxic stress exacerbate the rate of peripheral (intramuscular) failure $(2,23)$, largely due to a net increase in muscle fiber recruitment in order to match the increased anaerobic energy demands of a given mechanical output $(4,23,63)$. In the cold, human performance is also limited at the peripheral/intramuscular level $(47,69)$, partially caused by local vasoconstriction reducing venous washout of metabolic by-products in the active muscle (8). However vasoconstriction of active musculature is likely to be secondary to the progressive reductions in the absolute aerobic-mechanical efficiency caused by shivering $(35,69)$ and the coactivation of the antagonist muscles $(45,46)$.

While peripheral adaptations may partly explain environmental influences on exercise, both conscious and autonomic-inhibitory neural factors (i.e. central fatigue) $(5,17,33,64)$ have been recognized for their role in hot, cold and hypoxic performance decrements $(9,22,41)$. For example, suboptimal voluntary muscle activation (VA) independent of peripheral fatigue, has been reported under extreme heat stress $(39,65,67)$ and severe systemic hypoxemia $(22,37)$. Such acute reductions in VA have primarily been attributed to changes in cerebral temperature (44) and cerebral oxygenation (43) respectively. However, the identification of the involvement of numerous 'limiting' factors at the point 
121 of exhaustion has also highlighted the importance of psycho- and neurophysiological interactions

122 during exercise regulation, including cognitive-behavioral management of thermal and muscular

123 metabolic homeostasis $(5,13,32)$. In this regard, the afferent neural networks stemming from metabo-,

124 mechano-, thermo- and baroreceptors are likely crucial in integrating the cardiovascular and

125 mechanical (peripheral) adaptations under central control $(38,62)$.

126

127 Human performance during exposure to multifactorial environments is notably complex to investigate,

128 and literature examining inter-stressor interactions is sparse (66). Real world exposures to high-altitude

129 often consist of combinations of hypobaric hypoxia, solar radiation, winter clothing and cold ambient

130 temperatures $(30,31,66,68)$. In these complex situations, the effect of one stressor on performance

131 may be subject to change, simply due to the presence of another independent stressor. Such differential

132 influences can occur in three basic forms; additive, antagonistic and synergistic (31). Each term defines

133 a fundamental concept of inter-parameter interactions, which are most effectively represented using

134 example parameters 'A' and 'B'. Starting from an additive interaction, which mathematically can

135 either be the sum of absolute impacts:

136

Impact of 'A' and 'B' combined (\% reduction $)=$

Impact of 'A'(\% reduction $)+$ Impact of 'B' $(\%$ reduction $)$

137

138 Or the product of the relative impacts, expressed as:

139

Impact of 'A' and 'B' combined $(\%$ reduction $)=$

$$
\left[1-\left(1-\frac{\text { Impact of 'A' }(\% \text { reduction })}{100}\right) \cdot\left(1-\frac{\text { Impact of 'B' }(\% \text { reduction })}{100}\right)\right] \cdot 100
$$

140

141 The other options are that the combined effect is significantly larger (synergistic) than the sum of the

142 individual effects of each parameter:

143

$$
\begin{aligned}
& \text { Impact of 'A' and 'B' combined (\% reduction })> \\
& \text { Impact of ' } \mathrm{A} \text { ' }(\% \text { reduction })+\text { Impact of 'B' }(\% \text { reduction })
\end{aligned}
$$


145 Or smaller (antagonistic) than the sum of the individual effects of each parameter: Impact of 'A' and 'B' combined (\% reduction) <

Impact of 'A'(\% reduction $)+$ Impact of 'B' $(\%$ reduction $)$

147

148 An extreme expression of the antagonistic interaction might also include the complete abolition of one

149 or both stressors impact, and thereby the mathematical equivalent of the 'worst strain takes precedence'

150 up to a full 'strain nullification' respectively. Of the few studies that have investigated combined

151 temperature and hypoxia, Lloyd et al. (31) reported that forearm flexor fatigue increases additively

152 when hypoxia and mild cold are combined during repeated dynamic contractions. Likewise, Van

153 Cutsem et al. (68) and Aldous et al. (1) recently observed additive performance decrements when

154 combining hypoxia and a warm environment during 30 min self-paced cycling and an intermittent

155 soccer performance test respectively. In contrast however, Girard and Racinais (19) observed an

156 antagonistic interaction during a fixed intensity cycling $\left(66 \% \mathrm{VO}_{2 \max }\right)$ in combined moderate hypoxia

157 and mild heat stress. At present, the reasons for these varying observations are unclear.

159 As well as the natural occurrence of thermal and hypoxic stressors in combination $(31,66,68)$,

160 understanding interactions is fundamental to experimentally modelling how multiple physiological

161 strains integrate in their influence on - or regulation of - exercise intensity. Based on this, the present

162 study was primarily formulated to understand the causative factors behind different interaction

163 expressions, and thereby how interaction types can quantitatively define multifactorial integration

164 during exhaustive exercise. To achieve this, rates of peripheral and central fatigue development and

165 time to exhaustion (TTE) were examined across a variety of single and multi-stressor environments. By

166 examining both cold and heat stress combined with hypoxia (hypoxic-cold and hypoxic-heat

167 respectively), this study aimed to investigate both the influence of the individual stressor's mechanism

168 of impact (i.e. 'nature') as well as the individual stressor's magnitude of influence (i.e. 'impact

169 severity') on the interaction types expressed during combined exposure to simultaneous stressors.

170

171 Based on previous research, it was hypothesized that 1) short duration (40-mins) exposure to cold, heat

172 and hypoxia would each increase fatigue development and reduce TTE compared to thermoneutral

173 normoxia $(3,42,69) ; 2)$ the effect of short duration exposure to cold and hypoxia combined would be 
174 additive on TTE, central and peripheral fatigue development $(31)$; 3) the effect of short duration 175 exposure to heat and hypoxia would be antagonistic on TTE, central and peripheral fatigue

176 development (19); and 4) TTE and fatigue would be principally mediated by peripheral factors in 177 hypoxic-cold (31), while the contribution of central factors to TTE and fatigue would rise 178 synergistically in combined hypoxic-heat $(37,41)$.

179

180

181

182 183 184 185 186 187 188

\section{MATERIALS AND METHODS}

\section{Ethical approval}

This study was approved by Loughborough University Ethical Advisory Committee and was conducted in accordance with the World Medical Associations Declaration of Helsinki. Participants were provided with a detailed document explaining the risks and requirements of experimental protocol, prior to providing written informed consent. All participants conducted a health screening questionnaire prior to the start of the experiment.

\section{Subjects}

Nine healthy, moderately trained male volunteers participated in the study (mean \pm SD, height: $181 \pm$ $0.08 \mathrm{~cm}$, weight: $78.8 \pm 17.5 \mathrm{~kg}$, age: $22.1 \pm 2.1$ yrs., activity level: $4.8 \pm 1.2$ exercise bouts.wk ${ }^{-1}$, resting heart rate during 5-mins supine rest: $64 \pm 4$ b. $\mathrm{min}^{-1}$ ). All were right leg dominant with no previous history of cardiovascular, neurological and muscle debility. Participants were requested to preserve their normal exercise routines, but abstain from exercise, caffeine and alcohol 24 hrs. prior to each experimental session. The experiments were conducted in autumn (UK), presumably indicating little or no heat acclimation. Participants were not acclimated to hypoxia prior to participation in the experiment.

\section{General Study Overview}

Following initial familiarization, participants conducted six experimental sessions at three levels of environmental temperature ( $\left.\mathrm{T}_{\text {env }}\right)$ and two levels of fraction of inspired oxygen $\left(\mathrm{F}_{\mathrm{I}} \mathrm{O}_{2}\right)$ in an environmental chamber (T.I.S.S. Peak Performance, Series 2009 Climate Chambers). Specifically, the conditions included: 
HYP- COLD $\quad 5^{\circ} \mathrm{C} \mathrm{T}_{\text {env }} \& 50 \%$ rh in $0.125 \mathrm{~F}_{\mathrm{I}} \mathrm{O}_{2}$

205

COLD

$5^{\circ} \mathrm{C} \mathrm{T}_{\text {env }} \& 50 \%$ rh in $0.209 \mathrm{~F}_{\mathrm{I}} \mathrm{O}_{2}$

206

HYP- NEU

$23^{\circ} \mathrm{C} \mathrm{T}_{\text {env }} \& 50 \%$ rh in $0.125 \mathrm{~F}_{\mathrm{I}} \mathrm{O}_{2}$

207

NEU

$23^{\circ} \mathrm{C} \mathrm{T}_{\text {env }} \& 50 \%$ rh in $0.209 \mathrm{~F}_{\mathrm{I}} \mathrm{O}_{2}$

208

HYP- HEAT

$42^{\circ} \mathrm{C} \mathrm{T}_{\text {env }} \& 70 \%$ rh in $0.125 \mathrm{~F}_{\mathrm{I}} \mathrm{O}_{2}$

209

HEAT

$42^{\circ} \mathrm{C} \mathrm{T}_{\text {env }} \& 70 \%$ rh in $0.209 \mathrm{~F}_{\mathrm{I}} \mathrm{O}_{2}$

210

211 As a general overview of the experimental protocol, upon entering into the test conditions, participants

212 first performed an isometric assessment of neuromuscular function (PRE-REST). Following 40-mins

213 period of seated rest (REST), participants then performed a post-rest isometric assessment of

214 neuromuscular function (POST-REST). Subsequently, participants performed repeated bouts of

215 dynamic knee extension (DYN) at a fixed load (50.3 $\pm 11.1 \mathrm{~W} ; 60$ extensions.min ${ }^{-1} ; 80-140^{\circ}$ of knee

216 extension) until exhaustion (EXH). Every 110-s, dynamic exercise was interspersed with an isometric

217 neuromuscular test to calculate central and peripheral fatigue (ISO). Participants then continued

218 dynamic exercise until exhaustion in all conditions, completing a final isometric neuromuscular

219 function test at task failure (EXH). A complete schematic of the experimental protocol is provided in

220 Fig. 1.

221 [Insert Figure 1 here]

222

223

224 Familiarization sessions

225 Participants conducted at least two and up to four (dependent on the time necessary to ascertain an 226 appropriate workload; see below) preliminary sessions to familiarize with the experimental procedures

227 and requirements of the experiments. During these sessions, participants were accustomed to 228 performing brief maximal isometric voluntary contractions (MVC) with femoral nerve stimulation 229 (FNS; see procedural details below). In all sessions, this was followed by complete run through of the 230 experimental procedure, minus any rest periods (Fig. 1). To identify an appropriate workload for the 231 main experimental sessions, initial power was prescribed at a mental effort of two (light; Borg's CR-10 232 scale). Following this the change in mental effort was used to adjust power output, aiming to evoke 233 exhaustion in thermoneutral-normoxia (maximal on Borg's CR-10 scale) within 15 to 20 min of the 
235 (constant) workload based on each individual's performance in their previous familiarization session.

236 Participants then progressed to the main experimental sessions upon satisfactory completion of a full trial at a fixed workload that was observed to evoke exhaustion after no longer than $20 \mathrm{~min}$. The final

238 knee extension workload (different for each individual) was $50.3 \pm 11.1 \mathrm{~W}$. During familiarization, initial (fresh) MVC force was $940 \pm 156 \mathrm{~N}$ with an average co-efficient of variation (CV) in 3

240 successive trials of $3.81 \pm 1.4 \%$. Initial (fresh) resting potentiated twitch force was $384 \pm 53 \mathrm{~N}$ with an 241 average $\mathrm{CV}$ in 3 successive trials of $3.03 \pm 1.7 \%$.

242

\section{Main experimental sessions}

244 The main experimental procedure was the same across all conditions. Participants wore shorts and 245 socks for all conditions. Participants were instrumented with the temperature recording and 246 neuromuscular testing equipment, and muscle temperature $\left(T_{m}\right)$ was assessed prior to entering the 247 environmental chamber (see procedural details at section: temperature recording and oximetry). 248 Following this, participants sat in a custom-built knee extension dynamometer (inside the environmental chamber) and following potentiation (2-s plateau at 50, 50, 50, 75 and $90 \% \mathrm{MVC}$ ) of 250 the quadriceps they performed a pre-rest assessment of neuromuscular function (MVC of 2-s plateau duration with FNS, 3 times, with 30-s rest). Winter clothing was provided for pre-rest assessments in 252 cold conditions. Following this neuromuscular assessment, participants were then seated for 40-min 253 rest in the environmental chamber. During the rest periods, participants were instructed to maintain an 254 upright posture, with their arms relaxed by their side. After seated rest, $\mathrm{T}_{\mathrm{m}}$ was reassessed inside the 255 environmental chamber. Participants were then re-secured into the knee extension dynamometer 256 (details below), and following potentiation they performed a post-rest assessment of neuromuscular 257 function (MVC of 2-s plateau duration with FNS, 3 times, with 30-s rest). Following a final 90-s rest, 258 participants carried out dynamic knee extension (active concentric, passive eccentric) at a fixed 259 (constant) intensity $\left(50.3 \pm 11.1 \mathrm{~W} ; 60\right.$ extensions. $\min ^{-1} ; 80-140^{\circ}$ of knee extension) until exhaustion. 260 Each workload was specific to each individual and was selected based on their performance in the 261 familiarization trial. After every 110-secconds of dynamic knee extension exercise, the dynamometer was locked in position (knee joint angle $=100^{\circ}$ ), following which participants performed a single

263 MVC (2-s plateau duration with FNS) to quantify central and peripheral fatigue development (16). 

every 2-mintues of exercise. The locking of the knee joint angle was undertaken by a practiced experimenter. The average time from muscle relaxation after dynamic exercise to the start of MVC (i.e. the locking time) was $2.9 \pm 0.8$-s. Dynamic exercise resumed exactly 10 -s after relaxation from the previous bout. It should be noted that while a single MVC may be considered a less reliable measure of central and peripheral fatigue, it has the significant benefit of minimizing recovery time, thereby preventing the common underestimation of central and peripheral fatigue following dynamic exercise and at exhaustion (48).

272

273 Exhaustion was defined as either a) volitional cessation of exercise (i.e. exercise intolerance) or b) a 274 failure to maintain the required rate (e.g. 60 extensions. $\mathrm{min}^{-1}$ ) or range (e.g. $80-140^{\circ}$ of knee 275 extension) during three concentric knee extensor contractions in succession (i.e. task failure) (15).

\section{Application of the dynamic workload}

278 In this study a custom-built knee extension dynamometer was used. The equipment was designed based 279 on the work of Andersen et al (7) and Fulco et al (16). The initial seat and frame was taken from commercial knee extension equipment (GymanoElite Pro, UK). To apply a concentric contraction only load to the knee extensors, the weights system was replaced with an electromagnetically-braked flywheel (Angio V2, Lode, Groningen, Netherlands). The flywheel was powered using a noncompliant adjustable crank arm and chain driven gearbox. A locking mechanism was constructed to allow rapid changes between an isometric MVC (with FNS) and dynamic exercise $(15,16,48)$. A

285 visual-analogue scale was used to display current knee extension angle to the participants. The present range of movement (e.g. $80-140^{\circ}$ of knee extension) was selected based on piloting. This was 287 determined by an absence of antagonist stretching at extended ranges $\left(>150^{\circ}\right)$, with the aim of 288 minimizing monosynaptic co-activation of biceps femoris. The required rate (e.g. 60 extensions. $\min ^{-1}$ ) 289 was controlled using an audible metronome set to $2 \mathrm{~Hz}$ (a 'beep' for extension and flexion 290 respectively). The device was calibrated using dead weights of known mass (force) and goniometry 291 (range). During this experiment, the mean force transducer signal during each dynamic exercise bout 292 was strongly correlated $(\mathrm{r}=0.82)$ with the calculated power output (see below) across all participants. 
293 The CV in mean force transducer output during dynamic exercise across conditions was also small

294 (3.86\%), indicating equal and proportional workloads were successfully applied to the knee extensors.

295

296 Dynamometer set up

297 For all experiments, subjects were seated with a hip and knee joint angle of 90 and $100^{\circ}$ respectively.

298 The dynamometer was adjusted for each individual's femoral and tibial lengths, as well as their 299 popliteal to patella width. The right leg was secured to a force transducer (Tedea- Huntleigh, Model 300 615, Vishay Precision Group, California, USA) using an adjustable, non-compliant harness around 301 ankle malleolus. A layer of padding was applied to the ankle to protect against harness bruising/ 302 rubbing during the dynamic component of the exercise protocol. Participants were secured using a 303 waist belt system. Force data was visually displayed on a PC (DataLog software, Biometrics Ltd, UK) 304 via a Bluetooth wireless, 8 channel data logger (Miniature DataLog MWX8, Biometrics Ltd, UK). 305 Baseline noise was less than $0.5 \mathrm{~N}$ once ambient and force transducer temperature had stabilized. No 306 discernable (over and above baseline noise) differences were observed in force transducer sensitivity at 307 different $\mathrm{T}_{\text {env }}$.

308

309 Knee extension power output calculations

310 All power outputs were calculated from the kinetic energy measured on the flywheel, plus the power 311 required to move the lower leg, foot, force transducer and crank arm through $-20^{\circ}$ to $+40^{\circ}$ 312 (perpendicular to the floor) at an angular velocity of $2.0944 \mathrm{rad}^{-1}{ }^{-1}$ (i.e. movement through a range of $31380-140^{\circ}$ knee extension every $0.5 \mathrm{secs}$ ). Combined lower leg and foot weight were calculated as total 314 body weight $* 0.0592(12)$.

315

316 Since gravity alters the torque requirement at each circular section, a torque decay curve was calculated 317 for each $10^{\circ}$ segment moved. In this case, when the crank arm was perpendicular to the floor the 318 additional torque requirement equals zero; whereas this is equal to $100 \%$ when parallel to the floor. A 319 correction was then applied to the torque required at each $10^{\circ}$ segment to move the lower leg, foot, 320 force transducer and crank arm through the range used in this study, before calculating the total power 321 in watts (W) from flywheel power, angular velocity $\left(\right.$ rad.s $\left.\mathrm{s}^{-1}\right)$ and total corrected torque (N.m $\left.{ }^{-1}\right)$.

322 
324 Peripheral and central fatigue were calculated using the twitch interpolation technique during an MVC

325 (36). To this end, two superimposed twitches $\left(\mathrm{Q}_{\mathrm{tw}, \mathrm{sup}}\right)$ were evoked over the force plateau of each

326 MVC, each followed by two resting potentiated twitches $\left(\mathrm{Q}_{\mathrm{tw}, \mathrm{pot}}\right)$ 1-second after muscle relaxation. VA

327 (i.e. central fatigue) was calculated using the following equation $(32,41)$ : $\mathrm{VA}=\mathrm{MVC} /\left(\mathrm{MVC}+\mathrm{Q}_{\mathrm{tw}, \text { sup }}\right.$ ).

$328 \mathrm{Q}_{\mathrm{tw}, \mathrm{pot}}$ was used an index of the mechanical (contractile) properties of the muscle (i.e. peripheral

329 fatigue) $(4,32,53)$. The mean rate of force development (RFD) and mean rate of force relaxation

330 (RFR) were calculated for all $\mathrm{Q}_{\mathrm{tw}, \mathrm{pot}}(4)$.

331

332 In analysis, the values of the three MVC's pre- and post- rest were averaged and each set of $\mathrm{Q}_{\mathrm{tw}, \text { sup }}$ and

333 each set of $\mathrm{Q}_{\mathrm{tw}, \text { pot }}$ were averaged for each MVC. MVC force was taken as the average of two forces

334 sampled 1-ms prior to delivery of each $\mathrm{Q}_{\mathrm{tw}, \text { sup. }}$.

335

336 The femoral nerve was stimulated by two $0.2-\mathrm{ms}$ rectangular pulses spaced $10-\mathrm{ms}$ apart (i.e. doublet twitch), delivered using a high voltage simulator (max voltage $400 \mathrm{~V}$; Digitimer DS7AH,

338 Hertfordshire, UK) (14). The stimulator anode was placed in the femoral triangle and the cathode over the greater trochanter $(11,32,41,48)$. During familiarization the precise electrode placement was ascertained then marked with indelible ink. During familiarization the current necessary for supramaximal nerve depolarization was also calculated $(126 \pm 19 \mathrm{~mA})$, using progressive increases

342 until a plateau in the mechanical response of the muscle (i.e. $\left.\mathrm{Q}_{\mathrm{tw}, \mathrm{pot}}\right)$ was observed $(32,53)$.

343 Potentiation prior to both pre- and post-rest neuromuscular assessments (e.g. MVC of 2-s plateau 344 duration with FNS, 3 times, with 30-s rest) was ensured using a series of five incremental practice 345 contractions (2-s plateau at 50, 50, 50, 75 and 90\% MVC). Each neuromuscular test was conducted 15$346 \mathrm{~s}$ after potentiation. Subjects were encouraged moderately during all MVC's and all twitches were 347 delivered manually by the same experimenter.

\section{Temperature, heart rate and pulse oximetry}

350 Rectal temperature $\left(\mathrm{T}_{\text {core }}\right)$, skin temperature $\left(\mathrm{T}_{\mathrm{sk}}\right)$, heart rate $(\mathrm{HR})$ and oxygen saturation of peripheral 351 blood $\left(\mathrm{SpO}_{2}\right)$ were logged every 1 min from pre-exposure until exhaustion. Rectal temperature was measured $10-\mathrm{cm}$ beyond the anal sphincter using a flexible thermistor and squirrel data logger (Series 
2010, Grant International, UK). Skin temperature was measured on the forehead, shoulder, chest, right thigh, left thigh, right calf and left calf using wireless thermistors and in built memory (Ibutton, UK). Pulse oximetry was measured using a Nonin Pulse Oximeter (Nonin, US) attached to the ear lobe.

$\mathrm{T}_{\mathrm{m}}$ was measured at 1,2 and 3-cm depth using a solid needle thermocouple (Ellab, Denmark) inserted into vastus lateralis of both the exercising and non-exercising leg. Depth was corrected for adipose tissue, calculated using skin calipers over the insertion site. A strict sterility procedure was administered for all assessments. Data was collected prior to entering the environmental chamber (PRE-REST), after a 40-min seated rest period (POST-REST) and immediately following exhaustion (EXH) (Fig. 1). For simplicity, as well as to present the average gradient across the vastus lateralis muscle, $T_{\mathrm{m}}$ is displayed as a three depth mean $(1,2$ and $3-\mathrm{cm})$. Mean $\mathrm{T}_{\mathrm{sk}}$ was calculated using equal weighting from each of the seven measurement sites.

\section{Perceptual Ratings}

Immediately after every MVC intervention participants were asked to retrospectively rate their subjective sense of a) mental effort; b) leg muscle fatigue; and c) leg muscle pain for the previous bout of dynamic exercise. All participants were clearly instructed that sense of mental effort was the internal sense of effort expended, independent of all peripheral discomforts (10). All questions were answered by giving a rating on a modified Borg CR-10 scale (10). The specific questions were visibly printed above the scale and stated: a) 'what was your sense of leg effort i.e. how hard did you have to drive your leg'; b) 'what was your sense of fatigue in your exercising muscles'; and c) 'what was your sense of pain in your exercising muscles'.

\section{Statistical Analysis}

To examine the main effect of $\mathrm{F}_{1} \mathrm{O}_{2}$ (e.g. normoxia and hypoxia), and $\mathrm{T}_{\text {env }}$ (e.g. cold, neutral and heat) on all dependent variables, a two-way $\left(3 \times 2 ; \mathrm{T}_{\text {env }} \times \mathrm{F}_{\mathrm{I}} \mathrm{O}_{2}\right)$ repeated measures analysis of variance (ANOVA) was used. Two-way ANOVA's were conducted at time point's pre-rest, post-rest and exhaustion, as well on the rate of change $\left(\% \cdot \mathrm{min}^{-1}\right)$ between time points post-rest and exhaustion (i.e. between the start and end of the dynamic exercise). Given a main effect at any given time point (i.e. two-way ANOVA) will typically yield a significant interaction with time, for straightforwardness, the 
effect of time was not deemed necessary to assess using a three-way ANOVA. Significance was tested at a $95 \%$ confidence level $(\mathrm{p}<0.05)$. The Greenhouse- Geisser correction was applied when Mauchly's test of Sphericity was significant. When a significant $F$ ratio was observed for $T_{\text {env }}$, then pairwise comparisons (Bonferroni corrected) were conducted to assess the independent variance of cold or heat from neutral $\mathrm{T}_{\text {env }}$. When a significant stressor interaction $\left(\mathrm{T}_{\text {env }} \times \mathrm{F}_{1} \mathrm{O}_{2}\right)$ was observed in conjunction with significant main effects of heat, cold as well as $\mathrm{F}_{\mathrm{I}} \mathrm{O}_{2}$, an additional two-way $(2 \times 2$; HEAT or COLD vs NEU x $\mathrm{F}_{\mathrm{I}} \mathrm{O}_{2}$ ) repeated measures ANOVA was conducted to examine the interaction type expressed during hypoxic-heat and hypoxic-cold (31).

A different number of MVC's were performed by each participant across conditions (repeated every 110-s across varying exercise times; Fig. 1). It is therefore not appropriate to compare dependent variables across each MVC collection point. Consequently, non-linear regression analysis (dependent variable vs time) was used to define the temporal changes in central (VA) and peripheral fatigue $\left(\mathrm{Q}_{\mathrm{tw}, \mathrm{pot}}\right)$. Regression analyses were conducted on the individual data points (as opposed to group mean data) between start and end of the dynamic exercise, allowing overall mean curves, using the same time base, for each condition to be determined. Data are displayed as mean $\pm \mathrm{SD}$.

\section{RESULTS}

\section{Pre-rest measures}

Prior to the seated rest, there was no main effect of $\mathrm{T}_{\text {env }}$ or $\mathrm{F}_{\mathrm{l}} \mathrm{O}_{2}$ on $\mathrm{T}_{\text {core }}, \mathrm{T}_{\text {sk }}$, quadriceps $\mathrm{T}_{\mathrm{m}}$ (both legs), $\mathrm{SpO}_{2}, \mathrm{HR}, \mathrm{VA}, \mathrm{Q}_{\mathrm{tw}, \text { sup }}, \mathrm{Q}_{\mathrm{tw}, \mathrm{pot}}$, mean RFD or mean RFR. Average MVC force across the three pre-rest contractions was significantly lower during heat $(p=0.020)$ and cold $(p=0.003)$ compared with neutral conditions (-5 and $-7 \%$, respectively). While there were no significant pre-rest modulations in VA, VA did significantly $(\mathrm{p}<0.001)$ correlate with changes in MVC force $\left(\mathrm{R}^{2}=0.52\right.$ for condition normalized VA vs MVC). Moreover, the reductions in average MVC force were independent $(\mathrm{p}=$ $0.246)$ of any corresponding pre-experimental muscle fatigue $\left(\mathrm{R}^{2}=0.01\right.$ for condition normalized $\mathrm{Q}_{\mathrm{tw}, \text { pot }}$ vs MVC).

\section{[Insert Table 1 here]}

\section{Temperature, heart rate and pulse oximetry}


413 Table 2 shows the temperature recordings and pulse oximetry before and after the rest period, as well

414 as at exhaustion. $\mathrm{T}_{\text {core }}$ was unaffected by condition $(\mathrm{p}>0.2)$ except at the post-exercise time point

415 where $\mathrm{T}_{\text {core }}$ was $0.25^{\circ} \mathrm{C}$ higher $(\mathrm{p}=0.017)$ in the heated conditions compared to neutral. Conversely,

416 after the rest period, right leg quadriceps $\mathrm{T}_{\mathrm{m}}$ and mean $\mathrm{T}_{\text {sk }}$ decreased $(\mathrm{p}<0.002)$ by $3.8 \pm 1.8^{\circ} \mathrm{C}$ and 5.4

$417 \pm 0.6^{\circ} \mathrm{C}$ in cold conditions, increased $(\mathrm{p}<0.001)$ by $2.2 \pm 1.4^{\circ} \mathrm{C}$ and $5.1 \pm 1.1^{\circ} \mathrm{C}$ in heated conditions,

418 and decreased by $0.8 \pm 1.2^{\circ} \mathrm{C}$ and $0.3 \pm 0.5^{\circ} \mathrm{C}$ in neutral conditions. Rest in hypoxia did not affect

419 exercising quadriceps $\mathrm{T}_{\mathrm{m}}(\mathrm{p}=0.234)$; however mean $\mathrm{T}_{\mathrm{sk}}$ was marginally increased $(\mathrm{p}<0.001)$ by $0.4 \pm$

$420 \quad 0.5^{\circ} \mathrm{C}$ in hypoxic conditions. The dynamic exercise protocol increased exercising $\mathrm{T}_{\mathrm{m}}$ in all conditions;

421 however exercising $T_{m}$ remained significantly different $(p<0.001)$ in the same order across

422 environmental temperatures at exhaustion.

423

424 Across the whole exposure, mean $\mathrm{SpO}_{2}$ was significantly $(\mathrm{p}<0.001)$ reduced to $85 \pm 4 \%$ in hypoxia 425 compared to $99 \pm 1 \%$ in normoxia. $\mathrm{SpO}_{2}$ remained significantly $(\mathrm{p}<0.001)$ lower in hypoxia at 426 exhaustion. HR was significantly increased after the rest period in heated $(\mathrm{p}=0.002)$ and hypoxic $(\mathrm{p}=$ $4270.013)$ conditions.

428

429 There was no significant interaction $(p>0.1)$ between $F_{I} O_{2}$ and $T_{\text {env }}$ on any dependent variable 430 immediately after the rest period. At exhaustion however, there was a trend $(p=0.084)$ for synergistic 431 increases in $\mathrm{T}_{\mathrm{sk}}$, and a trend $(\mathrm{p}=0.062)$ for antagonistic decreases in $\mathrm{SpO}_{2}$, when hypoxia and cold 432 were combined. In addition, hypoxia significantly $(p=0.039)$ antagonized non-exercising $T_{m}$ in heated 433 conditions compared to neutral conditions; however this is most likely explained by the higher non434 exercising $\mathrm{T}_{\mathrm{m}}$ at the start of the exercise in the hypoxic-thermoneutral condition (Table 2).

435 [Insert Table 2 here]

\section{Post-rest neuromuscular measures}

438 After the rest period, there was no main effect of $\mathrm{F}_{\mathrm{I}} \mathrm{O}_{2}$ on MVC force, $\mathrm{VA}, \mathrm{Q}_{\mathrm{tw}, \text { sup }}, \mathrm{Q}_{\mathrm{tw}, \mathrm{pot}}$, mean RFD, 439 mean RFR, perceived mental effort, perceived leg fatigue or perceived leg pain. Additionally there was 440 no main effect of $\mathrm{T}_{\text {env }}$ on mean RFD, mean RFR, perceived mental effort, perceived leg fatigue or 441 perceived leg pain. However MVC force and VA were significantly reduced during heated exposures 
442 compared to neutral conditions $(\mathrm{p}=0.011$ and 0.006 respectively), suggesting participants displayed a

443 small degree of post-rest central fatigue in the heat (-4.0\% VA). While the main effect of $\mathrm{T}_{\mathrm{env}}$ on $\mathrm{Q}_{\mathrm{tw} \text {,pot }}$

444 was also significant $(\mathrm{p}=0.005)$, neither heat or cold were different from neutral $(\mathrm{p}>0.132)$, thus the

445 main effect was due to pairwise differences between heat and cold only $(\mathrm{p}=0.012)$.

446

447 Time to exhaustion

448 Fig. 2, Panel A shows the absolute TTE in seconds across all conditions, as well as the relative 449 reductions in TTE (percentage) caused by each individual stressor at each level of the other stressor 450 (Fig. 2 Table Insert). In response to dynamic exercise, independent exposure to hypoxia and to cold 451 reduced TTE by 505-s $(\mathrm{p}=0.002)$ and $190-\mathrm{s}(\mathrm{p}=0.006)$ respectively, from $915-\mathrm{s}$ in control 452 (thermoneutral-normoxic) conditions. During independent exposure to the heated condition, TTE was 453 significantly $(\mathrm{p}<0.001)$ reduced by 405-s (Fig. 2, Panel A).

454 [Insert Figure 2 here]

455

456 During combined hypoxic-cold, exercise time was reduced further (-589-s) compared to thermoneutral 457 normoxia $(-60 \pm 14 \%)$; however there was no significant interaction between stressors $(\mathrm{p}=0.198$; Fig. 458 2, Panel A). While the reduction in TTE in absolute terms did not visually appear additive of the two 459 stressors (suggesting antagonism) (Fig. 2, Panel A), the relative percentage reductions in TTE caused 460 by cold and hypoxia were similar irrespective whether this was during combined or individual stressor 461 exposure (Fig. 2, Table Insert). This suggests an additive relative effect (percentage reduction) when 462 cold and hypoxia are combined. Conversely, combined hypoxic-heat reduced exercise time by 609-s 463 compared to thermoneutral normoxia $(-63 \pm 13 \%)$, with a significant antagonistic interaction between 464 stressors $(\mathrm{p}=0.003)$. The relative influences of hypoxia and heat each were different in the presence of 465 the other stressor (Fig. 2, Table Insert) supporting a significant antagonism between heat and hypoxia 466 on TTE when combined. The interaction types expressed at group level varied slightly between 467 participants during both hypoxic-cold (6 additive, 2 antagonistic, 1 synergistic) and hypoxic-heat (2 468 additive, 7 antagonistic). 
470

471

472

473

474

475

476

477

478

479

480

481

482

483

484

485

486

487

488

489

490

491

492

493

494

495

496

497

498

499

In nearly all cases, volitional exercise intolerance occurred simultaneously with failure to maintain the required knee extension range (e.g. 80 to $140^{\circ}$ ) for three concentric knee extensor contractions in succession.

\section{Temporal change in central and peripheral fatigue}

Fig. 2, Panel B (peripheral fatigue/ $\mathrm{Q}_{\mathrm{tw}, \mathrm{pot}}$ ) and Table 3 (all other dependent variables) show rate of change in neuromuscular and perceptual variables between the start and end of the dynamic exercise (i.e. between post-rest and exhaustion). The rate of increase in peripheral fatigue $\left(\mathrm{Q}_{\mathrm{tw}, \mathrm{pot}}\right)$ was faster during independent exposure to cold $(p=0.004)$, heat $(p=0.006)$ and hypoxia $(p<0.001)$ compared to thermoneutral-normoxia (increases of $1.6 \pm 2.3,3.1 \pm 2.3$ and $4.9 \pm 2.7 \% \cdot \mathrm{min}^{-1}$ for cold, heat and hypoxia respectively). Moreover, the combined effects of hypoxia and cold as well as that of hypoxia and heat on peripheral fatigue rate were additive (increases of $7.6 \pm 3.2$ and $8.3 \pm 4.4 \% \cdot \mathrm{min}^{-1}$ ) with no significant interaction $(\mathrm{p}=0.525)$.

\section{[Insert Table 3 here]}

Interestingly, volitional (central) fatigue (VA) was largely unaffected at exhaustion (Table 1). Moreover, while the rates of change in VA were significantly greater in cold $(p=0.004)$ and heat $(p=$ 0.006), these were actually indicative of minor increases in VA (i.e. decreases in central fatigue). Also the rate of change in volitional fatigue was not affected by hypoxia $(p>0.37)$. When VA did decline (thermoneutral conditions only), the variance was less than $0.4 \% \cdot \min ^{-1}$ (Table 3 ).

\section{Central and peripheral contributions to exhaustion}

Fig. 3, Panels A, B, C and D illustrate the decline in $\mathrm{Q}_{\mathrm{tw}, \text { pot }}$ (increase in peripheral fatigue) over time and across conditions. Based on post-hoc observation, $\mathrm{Q}_{\mathrm{tw}, \mathrm{pot}}$ was fitted with a 2-order polynomial function $\left(\Delta \mathrm{Q}_{\mathrm{tw} \text {,pot }}(\%)=0.0011 \mathrm{x}^{2}-0.6617 \mathrm{x}-0.3017, \mathrm{R}^{2}=0.89\right.$, where $\mathrm{X}$ is percentage TTE). Fig. 4 ,

Panels A, B, C and D illustrate the change in VA (central fatigue) over time and across conditions. VA was fitted with a linear function (VA $=-0.0065 \mathrm{x}+93.383, \mathrm{R}^{2}=0.00$, where $\mathrm{X}$ is percentage TTE).

MVC force (an index of integrated fatigue) and perceptual exercise ratings (i.e. effort, fatigue and pain) each correlated with peripheral fatigue $\left(\mathrm{R}^{2}>0.79, \mathrm{p}<0.001\right)$ (Fig. 3, Panel E, F G and H), thus also 
500

501

502

503

504

505

506

507

508

509

510

511

512

513

514

515

516

517

518

519

520

521

522

523

524

525

526 In part confirmation of the second hypothesis, during combined exposure to hypoxia and cold, the

527 reductions in time to exhaustion were additive of the relative effects of hypoxia and cold

528 independently i.e. the fraction (percentage) decreases attributed to hypoxia and cold respectively were 529

following a 2-order polynomial function when expressed against time. Moreover, across conditions and over time, the perception of mental effort and quadriceps fatigue tracked an almost identical function to each other (perceived mental effort $=0.0003 \mathrm{x}^{2}+0.1252 \mathrm{x}+0.1411, \mathrm{R}^{2}=0.91$; perceived muscle fatigue $=0.0004 \mathrm{x}^{2}+0.128 \mathrm{x}+0.1349, \mathrm{R}^{2}=0.91$, where $\mathrm{X}$ is percentage TTE), resulting in similar values at the point of exhaustion. On the contrary, muscle pain was on average 1 point lower on the Borg CR-10 scale at any given time point (perceived muscle pain $=-0.0001 \mathrm{x}^{2}+0.0967 \mathrm{x}+0.0024, \mathrm{R}^{2}$ $=0.86$, where $\mathrm{X}$ is percentage TTE).

VA was largely unchanged over time and did not correlate in a meaningful way $\left(\mathrm{R}^{2}<.02\right)$ with the decline in MVC force $(p=0.013), Q_{t w, p o t}(p=0.407)$, or the increase in mental effort, perceived limb fatigue and perceived limb pain ( $p>0.39$ ) (Fig. 4, Panel E, F G and H).

\section{[Insert Fig. 3 here]}

\section{[Insert Fig. 4 here]}

\section{DISCUSSION}

The main focus of this study was to examine the effect of hypoxia and thermal stress (heat, neutral and cold environments) individually and combined, on the development of central and peripheral fatigue, as well as subsequent times to exhaustion. The results confirm the first hypothesis, that independent exposure to cold, heat and hypoxia each significantly reduced time to exhaustion with the effect increasing in that order (Fig. 2, Panel A); a finding related to changes in the rate of peripheral, not central, fatigue development (Fig. 2, Panel B; Fig. 3, Panel D). Since changes in peripheral fatigue occurred despite minimal increases in $\mathrm{T}_{\text {core }}$, the present data appear to support that thermoregulatory strain, and thereby muscle fatigue in the heat, is largely influenced the observed narrowing of the skin to core temperature gradient during high intensity exercise $(42,50,57,60)$ (Table 2$)$, though a direct effect of the raised skin and muscle temperature itself cannot be excluded.

\footnotetext{
similar during both combined and single stressor exposure (Fig. 2, Panel A). This differs from the
} 
530 findings of Lloyd et al. (31), where an absolute additive effect on fatigue was observed. The additive

531 reductions in times to exhaustion during hypoxic-cold (Fig. 2, Panel A) were also consistent with

532 additive rates of peripheral fatigue development (Fig. 2, Panel B; Fig. 3, Panel D) and an additive

533 progression in the perception of mental effort and leg muscle fatigue (Table 3; Fig. 3 Panels F and G

534 respectively). In contrast, combining moderate hypoxia with severe heat stress resulted in a significant

535 antagonistic interaction on both the absolute and relative reductions in time to exhaustion (Fig. 2) i.e.

536 the combined effect being significantly less than the sum of the individual effects. This confirms the

537 third hypothesis. Taking all observations together, the results suggest humans respond to severe and

538 simultaneous physiological strains based on a 'worst strain takes precedence' principle (details below).

539

540 Interestingly, the rate of increase in peripheral fatigue was strongly correlated with the decline in

541 isometric maximal voluntary force (a measure of integrated fatigue) and not accompanied by

542 substantial changes in voluntary activation (central fatigue) over time or across conditions. The results

543 suggest peripheral fatigue is likely the main driver behind faster neuromuscular fatigue (Fig. 3, Panel

544 E) and shorter times to exhaustion (Fig. 2) in the combined stressors tested presently, thus confirming

545 the additive hypothesis of hypoxic-cold, but opposing the hypoxic-heat hypothesis for central fatigue.

546

\section{Single stressor exposure to cold, heat and hypoxia}

548 Rate changes in peripheral (intermuscular/ mechanical) fatigue development (Fig. 2, Panel B; Fig. 3,

549 Panel D) are in line with previous reports using prolonged single-joint exercise under cold and hypoxic

550 stress $(15,28,45,67)$ as well as during high intensity exercise under heat stress $(20,40,50)$. In

551 hypoxia, an increase in peripheral fatigue development is commonly attributed to faster intra-muscle-

552 fiber metabolite production (e.g. inorganic phosphate, reactive oxygen species and hydrogen ion) (2,

553 24-26) due to a given mechanical work being performed at a higher relative aerobic strain (i.e.

$\left.554 \% \mathrm{VO}_{2 \max } \cdot \mathrm{W}^{-1}\right)$, with greater type II muscle fiber recruitment required to compensate inefficient oxygen

555 availability $(4,28,63)$. In the cold, an increase in muscle fatigue can be attributed to reductions in

556 active muscle perfusion (71), lowering both net oxygen delivery, and reducing muscle metabolite

557 washout (8). However in the cold, such factors are likely secondary to the reductions in aerobic-

558 mechanical efficiency (i.e. increase in $\mathrm{ml} \mathrm{O}_{2} \cdot \mathrm{min}^{-1} \cdot \mathrm{W}^{-1}$ ) (35) due to increases in antagonist co-

559 activation and increasing relative agonist muscle work $(45,46)$, which ultimately increases fiber 
560

561

562

563

564

565

566

567

568

569

570

571

572

573

574

575 It has been suggested that thermoregulatory strain (higher skin blood flow) is associated with the

576 observed narrowing of the skin to core temperature gradient $(42,57,60)$, as acutely observed in the

577 present study $(42,50,57,60)$ (Table 2). Thus an increase in muscle fatigue may have occurred because

578 higher HR and/or blood redistribution away from viscera was not able to entirely compensate the

579 requirement for both high skin and muscle blood flow. In this regard, it is pertinent to consider the

580 present exercise intensity and current test population, for whom the exercise workload was both high-

581

582

583

584

585

586

587

588

589

recruitment and thereby muscle (peripheral) fatigue $(4,23,63,69)$. While such mechanisms are likely responsible for the present findings, it should be noted that due to methodological constraints, changes in fiber recruitment and/or in co-activation were not directly addressed in this study (i.e. using EMG).

In the heat, dynamic exercise of isolated muscle groups has received less focus in the research compared to whole-body, multi-joint exercises. This is perhaps based on evidence suggesting that any reduction in central blood-volume (i.e. higher skin blood flow) is fully compensated for by increases in HR and redistribution of cardiac output away from non-essential vascular beds (e.g. renal, splanchnic, non-exercising muscle), leaving active muscle blood flow uncompromised during prolonged exercise of an isolated muscle group $(21,42,59)$. In contrast however, the present results report a $57 \%$ faster rate of peripheral fatigue development - and shorter TTE also - during high intensity, single-joint exercise in the heat compared to thermoneutral conditions (Fig. 2, Panel B; Fig. 3 Panel A, B, C, D); a finding in the absence of meaningful changes in both $\mathrm{T}_{\text {core }}$ (Table 2) and VA (central fatigue) (Table 1; Fig 4).

intensity and unsustainable in all environments, thus requiring high levels of muscle oxygen delivery

(Fig. 2, Panel A). By comparison, the maintained muscle blood flow observed by Savard et al. (59) employed an exercise workload that was sustainable for over 75-min. The increase in peripheral fatigue in the heat may also be related to the $\mathrm{Q}^{10}$ effect of $\mathrm{T}_{\mathrm{m}}$ on contractile efficiency (i.e. twitch fusion or oxygen uptake kinetics) $(23,27,54,61)$. At present however, the significance of the $\mathrm{Q}^{10}$ effect remains equivocal, especially during dynamic exercise, owing to the number of studies reporting an unchanged absolute oxygen consumption during localized and whole-body heat strain $(29,42)$.

\section{Combined exposure to thermal stress and hypoxia}


590 In this study, when thermal and hypoxic stressors were combined, TTE was substantially reduced,

591 compared with independent exposure to each stressor. Interestingly however, the stressor interaction

592 type differed between hypoxic-cold (relative addition) and hypoxic-heat (antagonistic) exposures (Fig.

593 2, Panel A). During combined hypoxic-cold, TTE reduced by a magnitude equal to the product of the

594 relative performance effects of each stressor individually. This is in partial support of previous studies

595 examining hypoxic-cold exposure during exercise of isolated muscle groups (31). However the present

596 results also differ in that the mean absolute reduction in performance (i.e. TTE) were not additive as

597 seen with previous additive interactions $(31,68)$. Supporting the findings of Lloyd et al. (31) however,

598 the present data do show that the influence of hypoxic-cold is mediated predominantly by peripheral

599 (intramuscular/ mechanical) factors, in the absence of alterations in central motor drive or fiber

600 recruitment (Fig. 3, 4). Thus, the present findings extend previous observations made in the smaller

601 forearm muscles, to demonstrate a relative additive effect in larger muscle groups at higher intensities,

602 as well as indicating that a faster development of peripheral fatigue (Fig. 2, Panel B) is a major

603 precursor to exhaustion in hypoxic-cold (Fig. 2, Panel A).

604

605 In contrast to hypoxic-cold, during combined hypoxia and heat, a significant antagonistic interaction

606 was observed on TTE. Thus, the relative effect magnitudes were reduced when hypoxia and heat were

607 in the presence of the other stressor (Fig. 2, Table Insert). Opposing the hypothesis, there was no

608 substantial influence of VA (central fatigue) on the decline in force with time (Table 1, Fig 4).

609 Therefore as with hypoxic-cold, the findings appeared to be primarily attributable to significant

610 increases in peripheral fatigue rate (Fig. 2, Panel B; Fig 3, Panel D).

611

612 The influence of individual stressor mechanisms and impact on the interaction between stressors

613 Lloyd et al. (31) suggested that when stressors with dissimilar mechanistic characteristics are

614 combined, an additive effect may be observed (e.g. cold and hypoxia); while combining stressors that

615 work through similar mechanisms, may result in an interactive effect (e.g. heat and hypoxia). This

616 suggestion was formulated on the basis that if two mechanisms work through similar physiological

617 pathways, there is a greater possibility for one stressor to influence another (i.e. cause an interaction).

618 Theoretically, this may elucidate contrasting interactions in this study, whereby oxygen transport

619 limitations in both heat and hypoxia interact during combined exposure (hypoxic-heat), while cold 
620

621

622

623

624

625

626

627

628

629

630

631

632

633

634

635

636

637

638

639

640

641

642

643

644 As well as characterizing multi-stressor environments, this novel paradigm may also reveal how not interact during combined (hypoxic-cold) exposure. However, the present findings should also be considered in light of two recent studies exploring combined hypoxia and thermal (warm/ heat) stress during whole-body exercise $(19,68)$. In these studies an additive effect on performance was reported by Van Cutsem et al. (68) during a self-paced cycling time trial; while an antagonistic interaction was observed by Girard and Racinais (19) during fixed, moderate intensity cycling to exhaustion. Because the nature of the stressors used in these studies were similar (warm-hypoxic and heat-hypoxic), additional reasons may need to be considered to explain the different interaction types observed during combined stressor exposures.

Another possible modulator of type of interaction may be the impact magnitude of the individual stressors' effects on performance. In this regard, individual stressors with a large influence on exercise capacity could antagonize when combined (19) (e.g. the present combination of moderate hypoxia and severe heat), while combined stressors that evoke milder performance reductions produce more additive effects $(1,31,68)$ (e.g. the present combination of moderate hypoxia and mild cold). This ultimately infers a maximum threshold for performance deterioration, whereby performance is only reduced by a specific magnitude before effects of a given stressor are fully antagonized i.e. the effect of one stressor is overruled or entirely cancelled out by the effect of the other. Based on present and past experimental data $(1,19,31,68)$, the magnitude of the stressors impact on performance likely provides a more suitable explanation for interaction type, compared to the pathway of influence (nature) of the two stressors being combined. Importantly, antagonism with increasing stressor impact indicates humans may respond to simultaneous and severe physiological strains based on a 'worst strain takes precedence' principle. multiple limiting factors can be imposed on exercise capacity, as well as clarifying the often

646 contrasting 'cardinal' limitations on exercise performance between studies. For example, in the heat, a 647 reduction in moderate-intensity exercise capacity is frequently associated with the concurrent increases

648 in core (spinal, visceral and cerebral) temperature $(19,42,68)$. Yet in the present study, increases in 649 skin and muscle $(13,32)$ temperature alone imposed severe limitations on high-intensity exercise 
650 performance (Table 2, Fig. 2). Based on the proposed paradigm of stressor antagonism (see above),

651 when simultaneously present, skin, muscle, spinal, visceral and cerebral temperature could each impose

652 their own task specific limitations on exercise capacity, however it is the factor with the greatest impact

653 magnitude that - for a given task - will progressively take precedence over all other factors.

654

655 Voluntary activation during brief exertions

656 The minimal changes in VA in the present study (Fig. 4) could be due to the small variation in $\mathrm{T}_{\text {core }}$

657 (Table 2), as well as the moderate level of systemic hypoxemia $\left(>80 \% \mathrm{SpO}_{2}\right)(22,37,39,65)$, both of

658 which should be acknowledged as limitations of the present study. The present findings may also be 659 attributable to the contractions used to measure VA being brief $(32,34,41)$. Brief contractions, 660 together with short ( $\sim 3-s)$ pauses in central motor drive prior to the MVC (49), reduce the impact of 661 afferent feedback from active, respiratory, cardiac and/or synergistic muscles to conscious moderations 662 in central motor drive (6), therefore facilitating VA to levels beyond what is possible at exhaustion. As 663 such, caution should be taken not to fully discount contributions from central factors to the present 664 reductions in TTE, which occurred during a sustained mental effort and with intact muscle sensory 665 feedback, prior to the brief MVC during which VA was measured.

666

667 It is important to note that an additional and unexpected finding in the present study was that MVC 668 force was slightly, though significantly, lower upon immediate exposure to the heated and cold 669 experimental conditions, compared with neutral $\mathrm{T}_{\text {env }}$. However, the small changes in MVC force did 670 correlate with changes in VA, and there were no corresponding changes in $\mathrm{Q}_{\mathrm{tw}, \mathrm{pot}}$ amplitude. This 671 indicates the pre-rest measures of MVC force may have been a psychophysiological response to acute 672 exposure to heat and cold (18), not an influence on the participants true ability to produce force or a 673 change in the force transducer sensitivity due to changes in $\mathrm{T}_{\text {env }}$.

674

\section{Perceptual responses to fatigue under environmental stress}

676 In the present study the rates of change in sensed mental effort, fatigue and pain were altered 677 proportionally to the environmental stressors influences on peripheral fatigue development (Fig, 3, 678 Panels F, G, and H). As such, the results appear to indicate that the rise in mental effort was in response 679 to the rise in actual and /or sensed muscle fatigue $(10,33)$, presumably via a progressive deactivation 
680

681

682

683

684

685

686

687

688

689

690

691

692

693

694

695

696

697

698

699

700

701

702

703

704

705

706

707

708

709 of muscle mechanoreceptive feedback for a given central command $(52,70)$ as well as the progressive activation of metaboreceptive feedback $(5,6,38,51)$. An important exception to this was in the rate of decline in leg muscle pain, which during cold was unchanged from neutral (Table 3) despite changes in TTE. This could be attributed to the attenuated excitability of sensory afferent nerves at lower $\mathrm{T}_{\mathrm{m}}(32$, $34,56,58)$.

\section{Conclusions, perspectives, context and significance of the research}

Exposure to real world extreme environments often consists of numerous environmental stressors and thereby multiple physiological strains. While recent studies conducted on small muscle groups (31) and whole-body exercise $(1,19,68)$ have begun to address how combined environmental stressors might influence exercise capacity, at present the basis for varied interaction types is unclear. By utilizing a mechanistic fatigue protocol across a variety of single and multi-stressor conditions, the roles of both stressor 'nature' and stressor 'impact magnitude' on the type of multi-stressor interaction expressed were examined.

Based on the conditions tested in the present study, combined exposure to moderate hypoxia and mild cold stress resulted in additive relative (percentage) reductions in times to exhaustion. In contrast, combined moderate hypoxia and severe heat stress resulted in a significant antagonistic interaction on time to exhaustion, where the effect of each stressor was attenuated in the presence of the other stressor. The decreases in time to exhaustion during both combined hypoxic-heat and combined hypoxic-cold were consistent with the increased rates of peripheral fatigue development, as well as a faster progression in perceived mental effort and muscle fatigue. Based on the present findings and previous research $(19,31,68)$, a novel principle of multifactorial integration is proposed; that the type of interaction between stressors is influenced by the impact magnitude of the individual stressors effect on exercise capacity, in which the greater the stressors impact, the greater the trend for one stressor to cancel out (nullify) the other. This is indicative of an 'antagonistic' or 'worst strain take precedence' model of multifactorial integration. 
1. Aldous JW, Chrismas BC, Akubat I, Dascombe B, Abt G, Taylor L. Hot and hypoxic environments inhibit simulated soccer performance and exacerbate performance decrements when combined. Front Physiol 6, 2015.

2. Allen DG, Lamb GD, Westerblad H. Skeletal Muscle Fatigue: Cellular Mechanisms. Physiol Rev 88: 287-332, 2008 .

3. Amann M, Calbet JAL. Convective oxygen transport and fatigue. J Appl Physiol 104: 86170, 2007.

4. Amann M, Romer LM, Pegelow DF, Jacques AJ, Hess CJ, Dempsey JA. Effects of arterial oxygen content on peripheral locomotor muscle fatigue. J Appl Physiol 101: 119-27, 2006.

5. Amann M, Sidhu SK, Weavil JC, Mangum TS, Venturelli M. Autonomic responses to exercise: Group III/IV muscle afferents and fatigue. Auton Neurosci 188: 19-23, 2015.

6. Amann M, Venturelli M, Ives SJ, McDaniel J, Layec G, Rossman MJ, Richardson RS. Peripheral fatigue limits endurance exercise via a sensory feedback-mediated reduction in spinal motoneuronal output. J Appl Physiol 115: 355-64, 2013.

7. Andersen P, Adams RP, Sjogaard G, Thorboe A, Saltin B. Dynamic knee extension as model for study of isolated exercising muscle in humans. J Appl Physiol 59: 1647-1653, 1985.

8. Blomstrand E, Bergh U, Essén-Gustavsson B, Ekblom B. Influence of low muscle temperature on muscle metabolism during intense dynamic exercise. Acta Physiol Scand 120: 229-36, 1984.

9. Cahill F, Kalmar JM, Pretorius T, Gardiner PF, Giesbrecht GG. Whole-body hypothermia has central and peripheral influences on elbow flexor performance. Exp Physiol 96: 528-38, 2011.

10. Christian RJ, Bishop DJ, Billaut F, Girard O. The role of sense of effort on self-selected cycling power output. Front Physiol 5: 115, 2014.

11. Christian RJ, Bishop DJ, Billaut F, Girard O. Peripheral fatigue is not critically regulated during maximal , intermittent, dynamic leg extensions. J Appl Physiol : 1063-1073, 2014.

12. Clauser CE, McConville JT, Young JW. Weight, Volume, and Center of Mass of Segments of the Human Body. Natl. Tech. Inf. Serv. (1969). doi: AMRL-TR- 69-70 (AD 710 622).

13. Flouris AD, Schlader ZJ. Human behavioral thermoregulation during exercise in the heat. Scand J Med Sci Sports 25: 52-64, 2015.

14. Folland JP, Williams AG. Methodological issues with the interpolated twitch technique. $J$ Electromyogr Kinesiol 17: 317-27, 2007.

15. Fulco CS, Lewis SF, Frykman PN, Boushel R, Smith S, Harman EA, Cymerman A, Pandolf KB, Charles S. Muscle fatigue and exhaustion during dynamic leg exercise in normoxia and hypobaric hypoxia. J Appl Physiol 81: 1891-1900, 1996.

16. Fulco CS, Lewis SF, Frykman PN, Boushel R, Smith S, Harman EA, Cymerman A, Pandolf KB. Quantitation of progressive muscle fatigue during dynamic leg exercise in humans. J Appl Physiol 79: 2154-2162, 1995.

17. Gandevia SC. Spinal and supraspinal factors in human muscle fatigue. Physiol Rev 81: 172589, 2001.

18. Gaoua N, Grantham J, Racinais S, EI Massioui F. Sensory displeasure reduces complex cognitive performance in the heat. J Environ Psychol 32: 158-163, 2012.

19. Girard O, Racinais $\mathbf{S}$. Combining heat stress and moderate hypoxia reduces cycling time to exhaustion without modifying neuromuscular fatigue characteristics. Eur J Appl Physiol 114: 1521-1532, 2014.

20. González-Alonso J, Calbet JAL. Reductions in Systemic and Skeletal Muscle Blood Flow and Oxygen Delivery Limit Maximal Aerobic Capacity in Humans. Circulation 107: 824-830, 2003.

21. González-Alonso J, Crandall CG, Johnson JM. The cardiovascular challenge of exercising 
in the heat. $J$ Physiol 586: 45-53, 2008.

22. Goodall S, Ross EZ, Romer LM. Effect of graded hypoxia on supraspinal contributions to fatigue with unilateral knee-extensor contractions. J Appl Physiol 109: 1842-51, 2010.

23. Grassi B, Rossiter HB, Zoladz JA. Skeletal muscle fatigue and decreased efficiency: two sides of the same coin? Exerc Sport Sci Rev 43: 75-83, 2015.

24. Haseler LJ, Hogan MC, Richardson RS. Skeletal muscle phosphocreatine recovery in exercise-trained humans is dependent on O2 availability. J Appl Physiol 86: 2013-2018, 1999.

25. Haseler LJ, Richardson RS, Videen JS, Hogan MC. Phosphocreatine hydrolysis during submaximal exercise: the effect of FIO2. J Appl Physiol 85: 1457-1463, 1998.

26. Hogan MC, Richardson RS, Haseler LJ. Human muscle performance and PCr hydrolysis with varied inspired oxygen fractions: a 31P-MRS study. J Appl Physiol 86: 1367-1373, 1999.

27. Kampmann B, Bröde P. Metabolic costs of physiological heat stress responses - Q10 coefficients relating oxygen consumption to body temperature. Extrem Physiol Med 4: A103, 2015.

28. Katayama K, Amann M, Pegelow DF, Jacques AJ, Dempsey JA. Effect of arterial oxygenation on quadriceps fatigability during isolated muscle exercise. Am J Physiol Regul Integr Comp Physiol 292: R1279-86, 2007.

29. Koga S, Shiojiri T, Kondo N, Barstow TJ. Effect of increased muscle temperature on oxygen uptake kinetics during exercise. J Appl Physiol 83: 1333-1338, 1997.

30. Levels K, de Koning J, Broekhuijzen I, Zwaan T, Foster C, Daanen HAM. Effects of radiant heat exposure on pacing pattern during a 15-km cycling time trial. J Sports Sci 32: 845$52,2014$.

31. Lloyd A, Hodder S, Havenith G. The interactive effect of cooling and hypoxia on forearm fatigue development. Eur J Appl Physiol 115: 2007-2018, 2015.

32. Lloyd A, Hodder S, Havenith G. The interaction between peripheral and central fatigue at different muscle temperatures during sustained isometric contractions. Am J Physiol Regul Integr Comp Physiol 309: R410-R420, 2015.

33. Marcora SM, Staiano W, Manning V. Mental fatigue impairs physical performance in humans. J Appl Physiol 106: 857-864, 2009.

34. Martin PG, Marino FE, Rattey J, Kay D, Cannon J. Reduced voluntary activation of human skeletal muscle during shortening and lengthening contractions in whole body hyperthermia. Exp Physiol 90: 225-36, 2005.

35. Mcardle D, Magel JR, Lesmes GR, Pechar GS. Metabolic and cardiovascular adjustment to work in air and water at 18, 25 and 33 ${ }^{\circ}$ C. J Appl Physiol 40: 85-90, 1976.

36. Merton PA. Voluntary Strength and Fatigue. J Physiol 1: 553-564, 1954.

37. Millet GY, Muthalib M, Jubeau M, Laursen PB, Nosaka K. Severe hypoxia affects exercise performance independently of afferent feedback and peripheral fatigue. J Appl Physiol 112: 1335-44, 2012.

38. Millet GY. Can neuromuscular fatigue explain running strategies and performance in ultramarathons?: The flush model. Sport Med 41: 489-506, 2011.

39. Morrison S, Sleivert GG, Cheung SS. Passive hyperthermia reduces voluntary activation and isometric force production. Eur J Appl Physiol 91: 729-36, 2004.

40. Nybo L, Jensen T, Nielsen B, González-Alonso J. Effects of marked hyperthermia with and without dehydration on VO2 kinetics during intense exercise. J Appl Physiol 90: 1057-1064, 2001 .

41. Nybo L, Nielsen B. Hyperthermia and central fatigue during prolonged exercise in humans. $J$ Appl Physiol 91: 1055-1060, 2001.

42. Nybo L, Rasmussen P, Sawka MN. Performance in the heat-physiological factors of importance for hyperthermia-induced fatigue. Compr Physiol 4: 657-689, 2014.

43. Nybo L, Rasmussen P. Inadequate cerebral oxygen delivery and central fatigue during strenuous exercise. Exerc Sport Sci Rev 35: 110-8, 2007. 
811 44. Nybo L. Brain temperature and exercise performance. Exp Physiol 97: 333-9, 2012.

812 45. Oksa J, Ducharme MB, Rintamäki H. Combined effect of repetitive work and cold on 813 muscle function and fatigue. J Appl Physiol 92: 354-361, 2002.

814 46. Oksa J, Rintamäki H, Rissanen S. Muscle performance and electromyogram activity of the

47. Oksa J. Neuromuscular performance limitations in cold. Int J Circumpolar Health 61: 154-62, 2002.

48. Pageaux B, Angius L, Hopker JG, Lepers R, Marcora SM. Central alterations of neuromuscular function and feedback from group III-IV muscle afferents following exhaustive high intensity one leg dynamic exercise. Am. J. Physiol. Regul. Integr. Comp. Physiol. (2015). doi: 10.1152/ajpregu.00280.2014.

49. Pageaux B, Marcora SM, Rozand V, Lepers R. Mental fatigue induced by prolonged selfregulation does not exacerbate central fatigue during subsequent whole-body endurance exercise. Front Hum Neurosci 9: 1-12, 2015.

50. Périard JD, Racinais S. Self-paced exercise in hot and cool conditions is associated with the maintenance of \%Vंo2peak within a narrow range. J Appl Physiol 118: 1258-1265, 2015.

51. Pollak KA, Swenson JD, Vanhaitsma TA, Hughen RW, Jo D, White AT, Light KC, Schweinhardt P, Amann M, Light AR. Exogenously applied muscle metabolites synergistically evoke sensations of muscle fatigue and pain in human subjects. Exp Physiol 99: 368-80, 2014.

52. Proske U. What is the role of muscle receptors in proprioception? Muscle and Nerve 31: 780787, 2005.

53. Racinais S, Gaoua N, Grantham J. Hyperthermia impairs short-term memory and peripheral motor drive transmission. J Physiol 586: 4751-4762, 2008.

54. Racinais S, Oksa J. Temperature and neuromuscular function. Scand J Med Sci Sports 20 Suppl 3: 1-18, 2010.

55. Ray CA, Gracey KH. Augmentation of exercise-induced muscle sympathetic nerve activity during muscle heating. J Appl Physiol 82: 1719-1733, 1997.

56. Ray CA, Hume KM, Gracey KH, Mahoney ET. Muscle cooling delays activation of the muscle metaboreflex in humans exercise humans. Am J Physiol Heart Circ Physiol 273: H2436-H2441, 1997.

57. Rowell LB. Human Cardiovascular Adjustments Exercise and Thermal Stress. Physiol Rev 54: 75-159, 1974.

58. Rutkove SB. Effects of temperature on neuromuscular electrophysiology. Muscle and Nerve 24: 867-882, 2001.

59. Savard GK, Nielsen B, Laszczynska J, Larsen BE, Saltin B. Muscle blood flow is not reduced in humans during moderate exercise and heat stress. J Appl Physiol 64: 649-657, 1988.

60. Sawka MN, Leon LR, Montain SJ, Sonna LA. Integrated physiological mechanisms of exercise performance, adaptation, and maladaptation to heat stress. Compr Physiol 1: 1883$1928,2011$.

61. Segal SS, Faulkner JA, White TP. Skeletal muscle fatigue in vitro is temperature dependent. J Appl Physiol 61: 660-665, 1986.

62. St Clair Gibson A, Noakes TD. Evidence for complex system integration and dynamic neural regulation of skeletal muscle recruitment during exercise in humans. Br J Sports Med 38: 797806, 2004.

63. Taylor AD, Bronks R, Smith P, Humphries B. Myoelectric evidence of peripheral muscle fatigue during exercise in severe hypoxia: some references to $\mathrm{m}$. vastus lateralis myosin heavy chain composition. Eur J Appl Physiol 75: 151-9, 1997.

64. Taylor JL, Todd G, Gandevia SC. Evidence for a supraspinal contributiuon to human muscle fatigue. Clin Exp Pharmacol Physiol 33: 400-405, 2006. 
65. Thomas MM, Cheung SS, Elder GC, Sleivert GG. Voluntary muscle activation is impaired by core temperature rather than local muscle temperature. J Appl Physiol 100: 1361-9, 2006.

66. Tipton M. A case for combined environmental stressor studies. Extrem Physiol Med 1: 7, 2012.

67. Todd G, Butler JE, Taylor JL, Gandevia SC. Hyperthermia: a failure of the motor cortex and the muscle. J Physiol 563: 621-31, 2005.

68. Van-Cutsem J, Pattyn N, Vissenaeken D, Dhondt G, De Pauw K, Tonoli C, Meeusen R, Roelands B. The influence of a mild thermal challenge and severe hypoxia on exercise performance and serum BDNF. Eur. J. Appl. Physiol. (2015). doi: 10.1007/s00421-015-3193$\mathrm{x}$.

69. Wakabayashi H, Oksa J, Tipton MJ. Exercise performance in acute and chronic cold exposure. J Phys Fit Sport Med 4: 177-185, 2015.

70. Winter JA, Allen TJ, Proske U. Muscle spindle signals combine with the sense of effort to indicate limb position. J Physiol 568: 1035-1046, 2005.

71. Yanagisawa O, Homma T, Okuwaki T, Shimao D, Takahashi H. Effects of cooling on human skin and skeletal muscle. Eur J Appl Physiol 100: 737-45, 2007.

\section{$\underline{\text { TABLE LEGENDS }}$}

Table 1: Neuromuscular function and perceptual exercise ratings before (PRE-REST) and after the rest period (POST-REST) and at exhaustion (EXH). From left to right the conditions are: hypoxic-cold (HYP-COLD), normoxic-cold (COLD), hypoxic-thermoneutral (HYP-NEU), normoxic-thermoneutral (NEU), hypoxic-heat (HYP-HEAT) and normoxic-heat (HEAT). MVC, maximal voluntary contraction; VA, voluntary muscle activation; $\mathrm{Q}_{\mathrm{tw}, \text { sup }}$, superimposed twitch force; $\mathrm{Q}_{\mathrm{tw}, \mathrm{pot}}$, resting potentiated twitch force; mean RFD, resting twitch mean rate of force development; mean RFR, resting twitch mean rate of relaxation. All PRE-REST and POST-REST values were averaged for the three maximal voluntary contractions (MVC). Each set of $\mathrm{Q}_{\mathrm{tw} \text {,sup }}$ and each set of $\mathrm{Q}_{\mathrm{tw} \text {,pot }}$ were averaged for each MVC. MVC force was taken as the average of two forces sampled 1-ms prior to delivery of each $\mathrm{Q}_{\mathrm{tw} \text {,sup. }}$ All data are presented as mean $\pm \mathrm{SD}$. ' $\mathrm{T}_{\mathrm{env}}$ ' indicates a main effect of environmental temperature. ' $\mathrm{O}_{2}$ ' indicates a main effect of environmental oxygen concentration. Where a main effect of $\mathrm{T}_{\text {env }}$ has been indicated, the significant pairwise comparisons are displayed in the subsequent brackets (e.g. HEAT, COLD).

Table 2: Temperature recordings and pulse oximetry before (PRE-REST) and after seated rest in the environmental conditions (POST-REST) and at exhaustion (EXH). From left to right the conditions are: hypoxic-cold (HYP-COLD), normoxic-cold (COLD), hypoxic-thermoneutral (HYP-NEU), normoxic-thermoneutral (NEU), hypoxic-heat (HYP-HEAT) and normoxic-heat (HEAT). $\mathrm{T}_{\text {core, }}$ core 
898

899

900

901

902

903

904

905

906

907

908

909

910

911

912

913

914

915

916

917

918

919

920

921

922

923

924

925

temperature; $\mathrm{T}_{\mathrm{m}}$, vastus lateralis muscle temperature; $\mathrm{T}_{\mathrm{sk}}$, skin temperature; HR, heart rate; $\mathrm{SpO}_{2}$, oxygen saturation of peripheral blood. $\mathrm{T}_{\mathrm{m}}$ is displayed as a three depth mean $(1,2$ and $3-\mathrm{cm})$. Mean $\mathrm{T}_{\mathrm{sk}}$ was calculated using equal weighting from each of the seven measurement sites. All data are presented as mean $\pm \mathrm{SD}$. ' $\mathrm{T}_{\text {env }}$ ' indicates a main effect of environmental temperature. ' $\mathrm{O}_{2}$ ' indicates a main effect of environmental oxygen concentration. Where a main effect of $\mathrm{T}_{\text {env }}$ has been indicated, the significant pairwise comparisons are displayed in the subsequent brackets (e.g. HEAT, COLD). Where a significant interaction $\left(\mathrm{T}_{\text {env }} \times \mathrm{F}_{\mathrm{I}} \mathrm{O}_{2}\right)$ has been indicated, the specific ANOVA interactions for hypoxiccold and hypoxic-heat are also displayed in the subsequent brackets (e.g. HEAT, COLD).

Table 3: Rate of change $\left(\% \cdot \mathrm{min}^{-1}\right)$ in neuromuscular and perceptual variables between the start (POSTREST) and end (exhaustion; EXH) of the exercise protocol. From left to right the conditions are: hypoxic-cold (HYP-COLD), normoxic-cold (COLD), hypoxic-thermoneutral (HYP-NEU), normoxicthermoneutral (NEU), hypoxic-heat (HYP-HEAT) and normoxic-heat (HEAT). MVC, maximal voluntary contraction; VA, voluntary muscle activation; $\mathrm{Q}_{\mathrm{tw}, \text { sup }}$, superimposed twitch force; mean RFD, resting twitch mean rate of force development; mean RFR, resting twitch mean rate of relaxation. Each set of $\mathrm{Q}_{\mathrm{tw} \text {,sup }}$ were averaged for each MVC. MVC force was taken as the average of two forces sampled 1-ms prior to delivery of each $\mathrm{Q}_{\mathrm{tw}, \mathrm{sup}}$. Pre and post rest neuromuscular assessments are not included in this table. All data represent a reduction over time (except where indicated by a + ), and are presented as mean $\pm \mathrm{SD}$. ' $\mathrm{T}_{\text {env }}$ ' indicates a main effect of environmental temperature. ' $\mathrm{O}_{2}$ ' indicates a main effect of environmental oxygen concentration. Where a main effect of $\mathrm{T}_{\text {env }}$ has been indicated, the pairwise comparisons are displayed in subsequent brackets (e.g. HEAT, COLD).

\section{FIGURE LEGENDS}

Figure 1: Overview of the experimental protocol. White boxes indicate the schematic overview of the experimental protocol. Grey boxes indicate the outcome measures. $\mathrm{T}_{\text {core }}$, rectal temperature; $\mathrm{T}_{\mathrm{m}}$, muscle temperature; $\mathrm{T}_{\mathrm{sk}}$, skin temperature; $\mathrm{MVC}$, maximal isometric voluntary contraction force of knee extensors; $\mathrm{HR}$, heart rate; $\mathrm{SpO}_{2}$, oxygen saturation of peripheral blood; PRE-REST, pre seated rest in the environmental conditions; REST, rest period; POST-REST, post seated rest in the environmental 
926

927

928

929

930

931

932

933

934

935

936

937

938

939

940

941

942

943

944

945

946

947

948

949

950 Figure 4: The contribution of voluntary activation (VA; central fatigue) to time to exhaustion (TTE) as

951 well as maximal voluntary contraction (MVC) force (integrated fatigue), sense of mental effort, sense

952 of leg fatigue and sense of leg pain. Panels A, B, C and D illustrate the change in VA over time and

953 across conditions. Based on post-hoc observations VA was fitted with a linear function. Panels E, F, G

954 and $\mathrm{H}$ show the relationship between central fatigue and MVC force, sense of mental effort, sense of 
955 leg fatigue and sense of leg pain using linear correlation (least squares method) with the reduction in

956 VA. Pre and post rest neuromuscular assessments are not included in this figure.

957 


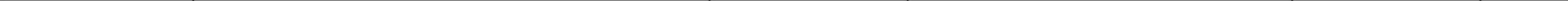




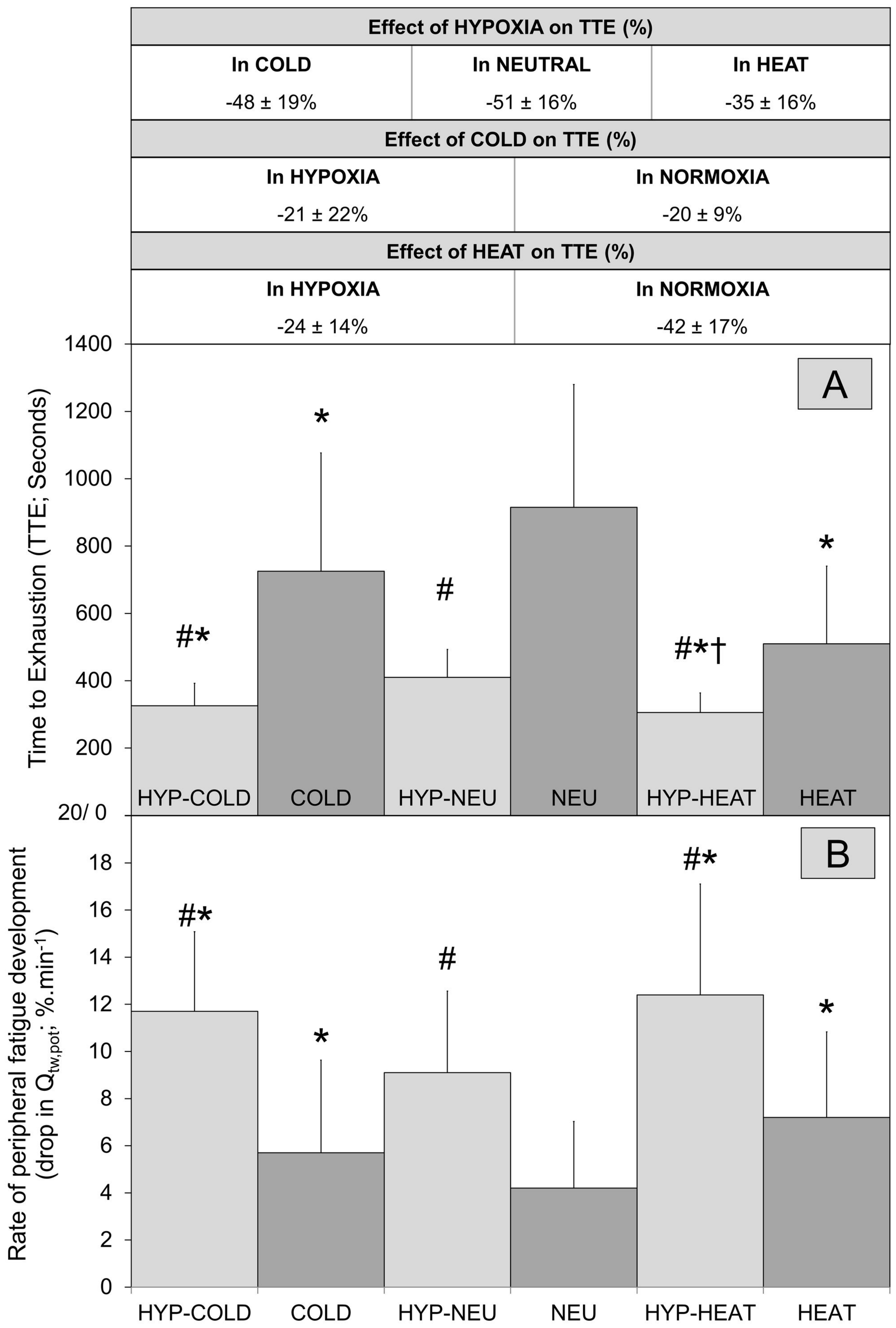




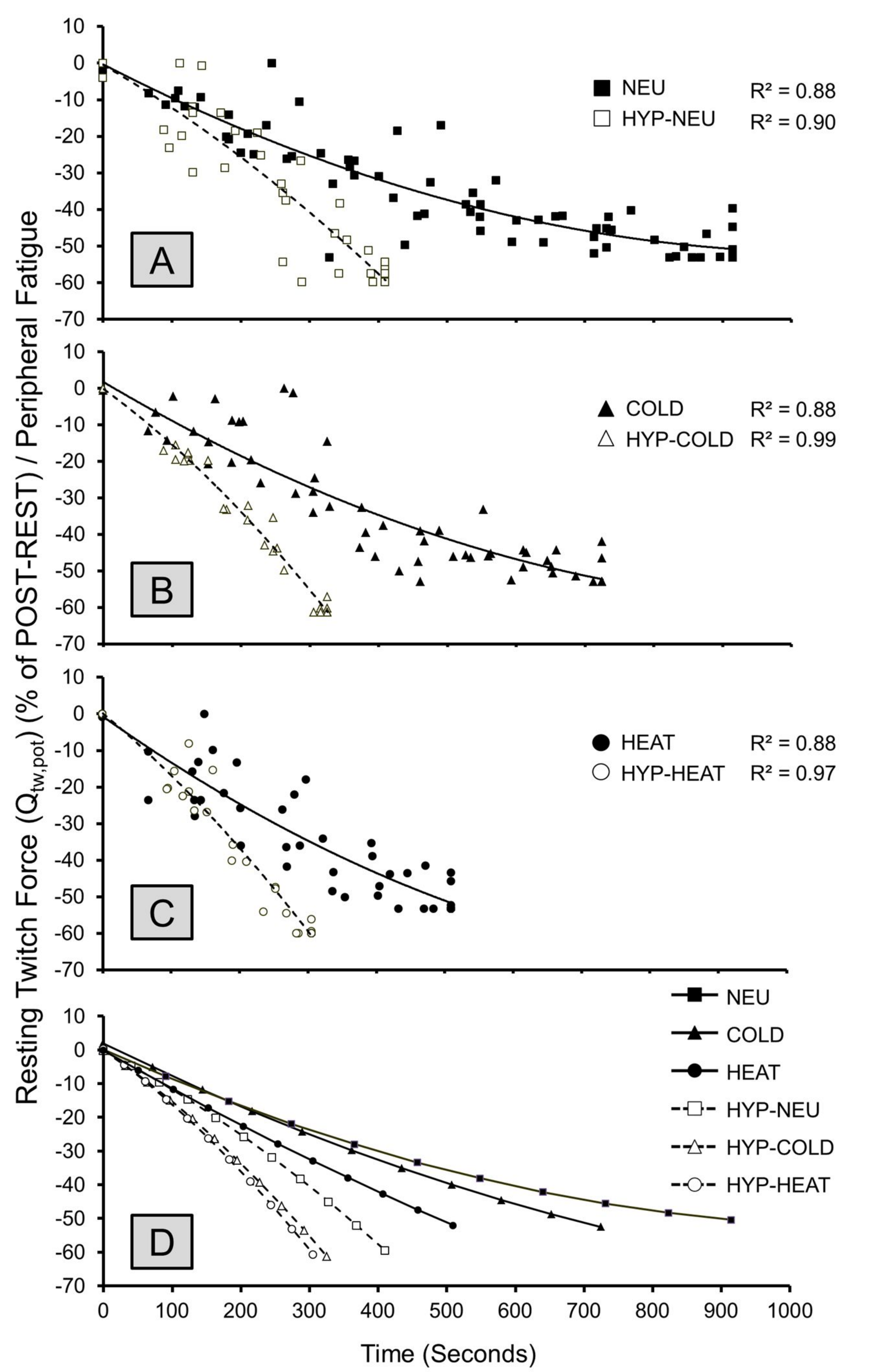

\begin{tabular}{lll}
$\widehat{E}^{-80} . \quad M V c$ vs $Q_{\mathrm{tw}, \mathrm{pot}}$ & $\mathrm{E}$ \\
\hline
\end{tabular}
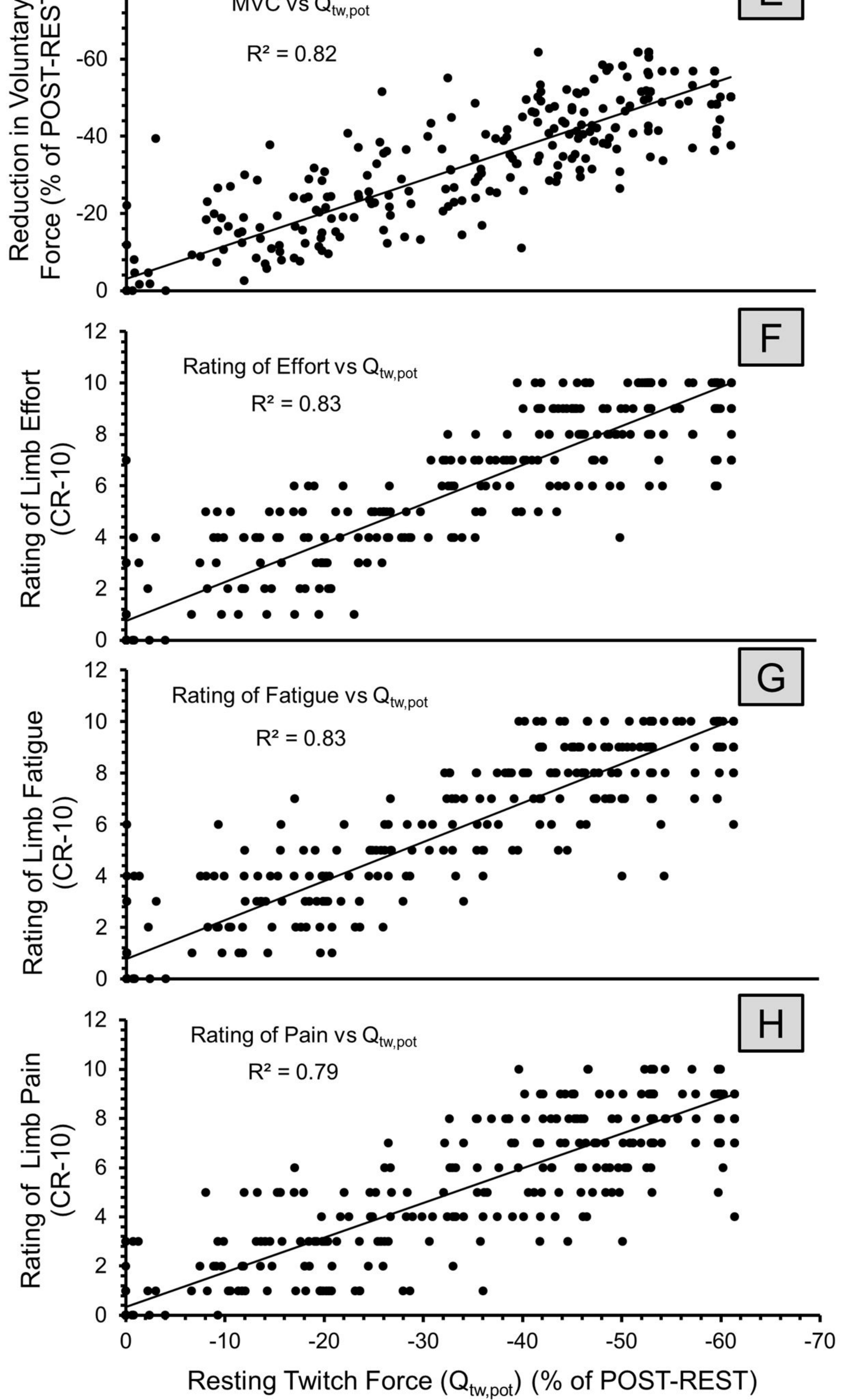


\begin{tabular}{|c|c|c|c|c|c|c|c|c|}
\hline Variable & Time Point & HYP-COLD & COLD & HYP-NEU & NEU & HYP-HEAT & HEAT & Main Effects \\
\hline \multirow{3}{*}{$\begin{array}{l}\text { MVC } \\
\text { Force } \\
(\mathrm{N})\end{array}$} & PRE-REST & $855 \pm 140$ & $835 \pm 172$ & $878 \pm 144$ & $915 \pm 199$ & $803 \pm 161$ & $861 \pm 194$ & $T_{\text {env }}($ HEAT, COLD $)$ \\
\hline & POST-REST & $869 \pm 139$ & $844 \pm 120$ & $903 \pm 131$ & $907 \pm 191$ & $770 \pm 142$ & $845 \pm 159$ & $\mathrm{~T}_{\text {env }}(\mathrm{HEAT})$ \\
\hline & EXH & $461 \pm 133$ & $436 \pm 89$ & $409 \pm 122$ & $395 \pm 100$ & $433 \pm 139$ & $436 \pm 113$ & $\mathrm{~T}_{\mathrm{env}}(\mathrm{COLD})$ \\
\hline \multirow{3}{*}{$\begin{array}{l}\text { VA } \\
(\%)\end{array}$} & PRE-REST & $93.3 \pm 3.0$ & $90.2 \pm 6.7$ & $91.3 \pm 4.9$ & $92.1 \pm 6.0$ & $89.3 \pm 6.6$ & $90.9 \pm 6.1$ & - \\
\hline & POST-REST & $93.2 \pm 3.1$ & $92.8 \pm 3.6$ & $93.7 \pm 3.2$ & $94.1 \pm 3.7$ & $88.8 \pm 7.6$ & $91.1 \pm 4.5$ & $\mathrm{~T}_{\text {env }}(\mathrm{HEAT})$ \\
\hline & EXH & $95.4 \pm 5.9$ & $91.7 \pm 11.5$ & $92.0 \pm 9.6$ & $91.0 \pm 9.5$ & $90.7 \pm 14.1$ & $91.9 \pm 5.5$ & $T_{\text {env }}$ \\
\hline \multirow{3}{*}{$\begin{array}{l}Q_{\mathrm{tw}, \text { sup }} \\
(\mathrm{N})\end{array}$} & PRE-REST & $79 \pm 34$ & $84 \pm 50$ & $81 \pm 43$ & $72 \pm 47$ & $90 \pm 46$ & $81 \pm 48$ & - \\
\hline & POST-REST & $60 \pm 25$ & $63 \pm 25$ & $59 \pm 30$ & $54 \pm 30$ & $93 \pm 59$ & $80 \pm 36$ & $T_{\text {env }}($ HEAT) \\
\hline & $\mathrm{EXH}$ & $18 \pm 20$ & $39 \pm 52$ & $36 \pm 48$ & $38 \pm 44$ & $40 \pm 59$ & $42 \pm 36$ & $\mathrm{O}_{2}$ \\
\hline \multirow{3}{*}{$\begin{array}{l}\mathrm{Q}_{\mathrm{tw}, \mathrm{pot}} \\
(\mathrm{N})\end{array}$} & PRE-REST & $339 \pm 45$ & $349 \pm 80$ & $368 \pm 90$ & $345 \pm 60$ & $350 \pm 68$ & $370 \pm 76$ & - \\
\hline & POST-REST & $322 \pm 49$ & $329 \pm 63$ & $337 \pm 63$ & $332 \pm 49$ & $344 \pm 61$ & $354 \pm 48$ & $\mathrm{~T}_{\text {env }}$ \\
\hline & $\mathrm{EXH}$ & $129 \pm 44$ & $167 \pm 80$ & $143 \pm 67$ & $166 \pm 68$ & $144 \pm 66$ & $177 \pm 80$ & $\mathrm{O}_{2}$ \\
\hline \multirow{3}{*}{$\begin{array}{c}\text { Mean RFD } \\
\left(\mathrm{N} \cdot \mathrm{ms}^{-1}\right)\end{array}$} & PRE-REST & $5.05 \pm 0.98$ & $5.08 \pm 0.83$ & $5.34 \pm 1.36$ & $4.92 \pm 0.60$ & $5.06 \pm 0.91$ & $5.33 \pm 0.86$ & - \\
\hline & POST-REST & $4.19 \pm 1.18$ & $4.28 \pm 1.20$ & $4.87 \pm 1.12$ & $3.91 \pm 1.18$ & $4.96 \pm 1.18$ & $4.52 \pm 1.03$ & - \\
\hline & EXH & $1.63 \pm 0.76$ & $2.38 \pm 1.26$ & $1.78 \pm 1.09$ & $2.48 \pm 1.28$ & $1.90 \pm 1.22$ & $2.45 \pm 1.39$ & $\mathrm{O}_{2}$ \\
\hline \multirow{3}{*}{$\begin{array}{c}\text { Mean RFR } \\
\left(\mathrm{N} \cdot \mathrm{ms}^{-1}\right)\end{array}$} & PRE-REST & $1.35 \pm 0.54$ & $1.43 \pm 0.50$ & $1.61 \pm 0.78$ & $1.46 \pm 0.41$ & $1.31 \pm 0.48$ & $1.58 \pm 0.58$ & - \\
\hline & POST-REST & $1.28 \pm 0.53$ & $1.32 \pm 0.27$ & $1.50 \pm 0.79$ & $1.56 \pm 0.82$ & $1.39 \pm 0.47$ & $1.47 \pm 0.57$ & - \\
\hline & $\mathrm{EXH}$ & $0.33 \pm 0.19$ & $0.65 \pm 0.42$ & $0.52 \pm 0.46$ & $0.69 \pm 0.50$ & $0.44 \pm 0.33$ & $0.65 \pm 0.50$ & $\mathrm{O}_{2}$ \\
\hline \multirow{3}{*}{$\begin{array}{l}\text { Sense of } \\
\text { Effort } \\
\text { (CR-10) }\end{array}$} & PRE-REST & - & - & - & - & - & - & - \\
\hline & POST-REST & $0.11 \pm 0.33$ & $0.00 \pm 0.00$ & $0.00 \pm 0.00$ & $0.00 \pm 0.00$ & $0.22 \pm 0.44$ & $0.00 \pm 0.00$ & - \\
\hline & $\mathrm{EXH}$ & $8.89 \pm 1.27$ & $9.44 \pm 0.73$ & $9.78 \pm 0.44$ & $9.78 \pm 0.44$ & $9.22 \pm 1.30$ & $9.78 \pm 0.44$ & $T_{\text {env }}$ \\
\hline \multirow{3}{*}{$\begin{array}{c}\text { Sense of Leg } \\
\text { Fatigue } \\
(\mathrm{CR}-10)\end{array}$} & PRE-REST & - & - & - & - & - & - & - \\
\hline & POST-REST & $0.11 \pm 0.33$ & $0.11 \pm 0.33$ & $0.11 \pm 0.33$ & $0.00 \pm 0.00$ & $0.22 \pm 0.44$ & $0.00 \pm 0.00$ & - \\
\hline & EXH & $9.11 \pm 1.26$ & $9.56 \pm 0.73$ & $9.56 \pm 0.73$ & $9.56 \pm 0.73$ & $9.44 \pm 0.73$ & $9.56 \pm 0.88$ & - \\
\hline \multirow{3}{*}{$\begin{array}{c}\text { Sense of Leg } \\
\text { Pain } \\
\text { (CR-10) }\end{array}$} & PRE-REST & - & - & - & - & - & - & - \\
\hline & POST-REST & $0.00 \pm 0.00$ & $0.00 \pm 0.00$ & $0.11 \pm 0.33$ & $0.00 \pm 0.00$ & $0.00 \pm 0.00$ & $0.00 \pm 0.00$ & - \\
\hline & $\mathrm{EXH}$ & $8.00 \pm 0.73$ & $8.56 \pm 0.88$ & $9.00 \pm 0.87$ & $9.00 \pm 1.12$ & $9.11 \pm 0.60$ & $9.00 \pm 0.87$ & $T_{\text {env }}$ \\
\hline
\end{tabular}




\begin{tabular}{|c|c|c|c|c|c|c|c|c|}
\hline Variable & Time Point & HYP-COLD & COLD & HYP-NEU & NEU & HYP-HEAT & HEAT & Main Effects \\
\hline \multirow{3}{*}{$\begin{array}{l}\text { Rectal } \mathrm{T}_{\text {core }} \\
\left({ }^{\circ} \mathrm{C}\right)\end{array}$} & PRE-REST & $37.24 \pm 0.26$ & $37.26 \pm 0.34$ & $37.31 \pm 0.24$ & $37.36 \pm 0.16$ & $37.28 \pm 0.23$ & $37.28 \pm 0.37$ & - \\
\hline & $\begin{array}{l}\text { POST-REST } \\
(\Delta)\end{array}$ & $-0.13 \pm 0.20$ & $0.00 \pm 0.58$ & $-0.18 \pm 0.17$ & $-0.15 \pm 0.10$ & $0.02 \pm 0.17$ & $0.03 \pm 0.18$ & - \\
\hline & $\mathrm{EXH}(\Delta)$ & $-0.22 \pm 0.23$ & $-0.08 \pm 0.64$ & $-0.14 \pm 0.16$ & $-0.09 \pm 0.11$ & $0.12 \pm 0.18$ & $0.14 \pm 0.16$ & $\mathrm{~T}_{\text {env }}(\mathrm{HEAT})$ \\
\hline \multirow{3}{*}{$\begin{array}{l}\text { Whole Body } \\
\mathrm{T}_{\text {sk }}\left({ }^{\circ} \mathrm{C}\right)\end{array}$} & PRE-REST & $32.1 \pm 0.9$ & $31.9 \pm 0.9$ & $32.3 \pm 0.7$ & $31.9 \pm 0.8$ & $31.8 \pm 1.0$ & $31.9 \pm 1.3$ & - \\
\hline & POST-REST & $26.8 \pm 0.9$ & $26.3 \pm 0.7$ & $32.4 \pm 0.7$ & $31.9 \pm 0.7$ & $37.0 \pm 0.2$ & $36.8 \pm 0.3$ & $\begin{array}{c}\mathrm{T}_{\text {env }} \text { (HEAT, COLD); } \\
\mathrm{O}_{2}\end{array}$ \\
\hline & $\mathrm{EXH}$ & $26.6 \pm 1.0$ & $25.9 \pm 0.7$ & $32.6 \pm 0.6$ & $32.5 \pm 0.8$ & $37.1 \pm 0.3$ & $37.1 \pm 0.4$ & $\begin{array}{c}\mathrm{T}_{\text {env }}(\mathrm{HEAT}, \mathrm{COLD}) \\
\mathrm{O}_{2} ; \mathrm{T}_{\text {env }} \times \mathrm{O}_{2}\end{array}$ \\
\hline \multirow{3}{*}{$\begin{array}{l}\text { Exercising } \\
\text { Vastus } \\
\text { Lateralis } \mathrm{T}_{\mathrm{m}} \\
\quad\left({ }^{\circ} \mathrm{C}\right)\end{array}$} & PRE-REST & $34.1 \pm 0.6$ & $34.1 \pm 1.4$ & $33.8 \pm 0.9$ & $34.4 \pm 1.3$ & $33.7 \pm 1.7$ & $34.4 \pm 0.8$ & $\mathrm{O}_{2}$ \\
\hline & POST-REST & $30.5 \pm 1.9$ & $30.2 \pm 1.5$ & $33.5 \pm 0.7$ & $33.1 \pm 1.0$ & $36.2 \pm 0.5$ & $36.4 \pm 0.3$ & $\mathrm{~T}_{\mathrm{env}}$ (HEAT, COLD) \\
\hline & $\mathrm{EXH}$ & $34.4 \pm 1.2$ & $34.2 \pm 1.8$ & $36.2 \pm 0.5$ & $36.3 \pm 0.6$ & $37.6 \pm 0.5$ & $37.9 \pm 0.5$ & $\mathrm{~T}_{\mathrm{env}}(\mathrm{HEAT}, \mathrm{COLD})$ \\
\hline \multirow{3}{*}{$\begin{array}{l}\text { Non- } \\
\text { Exercising } \\
\text { Vastus } \\
\text { Lateralis } \mathrm{T}_{\mathrm{m}} \\
\quad\left({ }^{\circ} \mathrm{C}\right)\end{array}$} & PRE-REST & $34.3 \pm 0.6$ & $33.9 \pm 1.0$ & $33.7 \pm 0.8$ & $34.5 \pm 1.0$ & $33.7 \pm 1.3$ & $34.1 \pm 0.6$ & - \\
\hline & POST-REST & $30.6 \pm 1.3$ & $29.9 \pm 1.6$ & $33.3 \pm 0.8$ & $32.7 \pm 1.3$ & $35.9 \pm 0.5$ & $36.0 \pm 0.4$ & $\mathrm{~T}_{\text {env }}$ (HEAT, COLD) \\
\hline & $\mathrm{EXH}$ & $29.1 \pm 1.6$ & $28.0 \pm 1.6$ & $32.9 \pm 0.9$ & $32.2 \pm 1.3$ & $36.1 \pm 0.5$ & $36.3 \pm 0.6$ & $\begin{array}{l}\mathrm{T}_{\text {env }} \text { (HEAT, COLD); } \\
\mathrm{O}_{2} ; \mathrm{T}_{\text {env }} \times \mathrm{O}_{2}(\text { HEAT) }\end{array}$ \\
\hline \multirow{3}{*}{$\mathrm{SpO}_{2}(\%)$} & PRE-REST & $99.7 \pm 0.7$ & $99.7 \pm 0.5$ & $99.6 \pm 0.7$ & $99.4 \pm 0.7$ & $99.6 \pm 0.7$ & $99.7 \pm 0.7$ & - \\
\hline & POST-REST & $83.1 \pm 2.8$ & $99.2 \pm 0.7$ & $86.4 \pm 4.6$ & $98.8 \pm 1.0$ & $87.7 \pm 4.8$ & $98.4 \pm 0.5$ & $\mathrm{O}_{2}$ \\
\hline & $\mathrm{EXH}$ & $87.3 \pm 5.6$ & $96.8 \pm 4.6$ & $90.3 \pm 4.4$ & $96.6 \pm 3.9$ & $92.4 \pm 3.7$ & $99.2 \pm 0.4$ & $\begin{array}{l}\mathrm{T}_{\text {env }} ; \mathrm{O}_{2} \\
\mathrm{~T}_{\mathrm{env}} \times \mathrm{O}_{2}\end{array}$ \\
\hline \multirow{3}{*}{$\operatorname{HR}\left(\right.$ b. $\left.\min ^{-1}\right)$} & PRE-REST & $70.6 \pm 6.1$ & $73.0 \pm 7.3$ & $72.6 \pm 11.2$ & $72.0 \pm 4.9$ & $72.7 \pm 7.2$ & $72.7 \pm 9.0$ & - \\
\hline & POST-REST & $84.3 \pm 15.2$ & $81.1 \pm 11.0$ & $94.3 \pm 11.8$ & $78.9 \pm 9.9$ & $108.8 \pm 12.1$ & $100.6 \pm 15.1$ & $\mathrm{~T}_{\mathrm{env}}(\mathrm{HEAT}) ; \mathrm{O}_{2}$ \\
\hline & EXH & $116.4 \pm 14.6$ & $124.2 \pm 18.9$ & $141.0 \pm 30.6$ & $132.6 \pm 28.1$ & $149.3 \pm 11.5$ & $157.8 \pm 13.7$ & $\mathrm{~T}_{\mathrm{env}}$ \\
\hline
\end{tabular}




\begin{tabular}{|c|c|c|c|c|c|c|c|c|}
\hline Variable & Time Point & HYP-COLD & COLD & HYP-NEU & NEU & HYP-HEAT & HEAT & Main Effects \\
\hline $\begin{array}{c}\text { MVC } \\
\text { Force } \\
\left(\% \cdot \text { min }^{-1}\right)\end{array}$ & $\begin{array}{l}\text { POST-REST } \\
\text { to EXH Rate } \\
\text { of Change }\end{array}$ & $9.03 \pm 2.86$ & $4.71 \pm 2.07$ & $8.39 \pm 2.63$ & $4.23 \pm 2.20$ & $8.86 \pm 2.80$ & $6.42 \pm 2.38$ & $\mathrm{~T}_{\text {env }}, \mathrm{O}_{2}$ \\
\hline $\begin{array}{c}\text { VA } \\
\left(\% \cdot \text { min }^{-1}\right)\end{array}$ & $\begin{array}{l}\text { POST-REST } \\
\text { to EXH Rate } \\
\text { of Change }\end{array}$ & $+0.45 \pm 0.90$ & $+0.13 \pm 0.90$ & $0.16 \pm 1.13$ & $0.16 \pm 0.53$ & $+0.51 \pm 1.54$ & $+0.31 \pm 0.69$ & $\mathrm{~T}_{\text {env }}$ (HEAT, COLD) \\
\hline$\underset{\left(\% \cdot \mathrm{min}^{-1}\right)}{\mathrm{Q}_{\mathrm{tw}, \mathrm{sup}}}$ & $\begin{array}{l}\text { POST-REST } \\
\text { to EXH Rate } \\
\text { of Change }\end{array}$ & $14.07 \pm 6.14$ & $6.04 \pm 8.06$ & $7.33 \pm 7.40$ & $2.68 \pm 5.03$ & $13.95 \pm 6.80$ & $7.44 \pm 7.49$ & $\begin{array}{l}T_{\text {env }}(H E A T, \\
\text { COLD), } O_{2}\end{array}$ \\
\hline $\begin{array}{l}\text { Mean RFD } \\
\left(\% \cdot \mathrm{min}^{-1}\right)\end{array}$ & $\begin{array}{l}\text { POST-REST } \\
\text { to EXH Rate } \\
\text { of Change }\end{array}$ & $11.99 \pm 4.33$ & $5.23 \pm 4.43$ & $9.54 \pm 5.18$ & $2.78 \pm 5.01$ & $12.97 \pm 5.70$ & $6.52 \pm 4.87$ & $\mathrm{~T}_{\text {env }}(\mathrm{HEAT}), \mathrm{O}_{2}$ \\
\hline $\begin{array}{c}\text { Mean RFR } \\
\left(\% \cdot \text { min }^{-1}\right)\end{array}$ & $\begin{array}{l}\text { POST-REST } \\
\text { to EXH Rate } \\
\text { of Change }\end{array}$ & $14.40 \pm 4.36$ & $6.28 \pm 5.25$ & $10.38 \pm 3.35$ & $4.56 \pm 3.29$ & $13.93 \pm 5.19$ & $7.69 \pm 4.54$ & $\begin{array}{c}\left.\mathrm{T}_{\text {env }} \text { (HEAT, COLD }\right) \\
, \mathrm{O}_{2}\end{array}$ \\
\hline $\begin{array}{l}\text { Sense of } \\
\text { Effort } \\
\left(\% \cdot \text { min }^{-1}\right)\end{array}$ & $\begin{array}{l}\text { POST-REST } \\
\text { to EXH Rate } \\
\text { of Change }\end{array}$ & $16.76 \pm 4.39$ & $9.63 \pm 4.76$ & $14.86 \pm 3.25$ & $7.40 \pm 3.09$ & $17.93 \pm 2.94$ & $13.11 \pm 4.18$ & $\mathrm{~T}_{\text {env }}(\mathrm{HEAT}), \mathrm{O}_{2}$ \\
\hline $\begin{array}{l}\text { Sense of Leg } \\
\text { Fatigue } \\
\left(\% \cdot \text { min }^{-1}\right)\end{array}$ & $\begin{array}{l}\text { POST-REST } \\
\text { to EXH Rate } \\
\text { of Change }\end{array}$ & $17.18 \pm 4.38$ & $9.69 \pm 4.84$ & $14.33 \pm 3.25$ & $7.24 \pm 3.14$ & $18.52 \pm 3.00$ & $12.76 \pm 4.12$ & $\begin{array}{l}T_{\text {env }}(H E A T, \\
\text { COLD), } O_{2}\end{array}$ \\
\hline $\begin{array}{c}\text { Sense of Leg } \\
\text { Pain } \\
\left(\% \cdot \text { min }^{-1}\right)\end{array}$ & $\begin{array}{l}\text { POST-REST } \\
\text { to EXH Rate } \\
\text { of Change }\end{array}$ & $15.48 \pm 5.36$ & $8.84 \pm 4.53$ & $13.55 \pm 3.21$ & $7.01 \pm 3.41$ & $18.43 \pm 3.50$ & $12.35 \pm 4.68$ & $\mathrm{~T}_{\text {env }}(\mathrm{HEAT}), \mathrm{O}_{2}$ \\
\hline
\end{tabular}

